\title{
Characterizing PID Controllers for Linear Time-Delay Systems: A Parameter-Space Approach
}

\author{
Xu-Guang Li, Silviu-Iulian Niculescu, Fellow, IEEE, Jun-Xiu Chen, and Tianyou Chai, Fellow, IEEE
}

\begin{abstract}
We focus on the proportional-integral-derivative (PID) controller design for linear time-delay systems. All the controller gains $\left(k_{P}, k_{I}\right.$, and $\left.k_{D}\right)$ and the delay $(\tau)$ are treated as free parameters and no particular constraints are imposed on the controlled plants. Such a problem (involving totally four free parameters) is of theoretical as well as practical importance, but, to the best of the authors' knowledge, it has not been fully explored. First, we will develop an algebraic algorithm to solve the complete stability problem w.r.t. $\tau$. Consequently, for any given PID controller vector $\left(k_{P}, k_{I}, k_{D}\right)$, the distribution of $N U(\tau)(N U(\tau)$ denotes the number of characteristic roots in the right-half plane, as a function of $\tau$ ) can be accurately obtained and the exhaustive stability range of $\tau$ may be automatically calculated. Next, a global understanding of the distribution of $N U(\tau)$ over the whole $\left(k_{P}, k_{I}, k_{D}\right)$-space may be achieved and all structural changes regarding the $N U(\tau)$ distribution can be analytically determined. To achieve such a goal, a complete positive real root classification (for some appropriate auxiliary characteristic equation) will be explicitly proposed. Finally, we will give a new methodology, a new parameter-space approach, for determining the stability set in the $\left(k_{P}, k_{I}, k_{D}, \tau\right)$-space.
\end{abstract}

Index Terms-Time-delay systems, proportional-integralderivative (PID) controllers, complete stability analysis, asymptotic behavior analysis, complete positive real root classification.

\section{INTRODUCTION}

The proportional-integral-derivative (PID) controller design for time-delay systems is a classical problem (see e.g., [29]). On one hand, PID controllers are used in more than $95 \%$ of industrial processes [2]. On the other hand, time-delay phenomena exist in almost all practical control system (see e.g., [7] and [20]).

Consider a SISO controlled plant with the transfer function:

$$
H_{0}(\lambda)=\frac{H_{\mathrm{N}}(\lambda)}{H_{\mathrm{D}}(\lambda)},
$$

where $H_{\mathrm{D}}(\lambda)$ and $H_{\mathrm{N}}(\lambda)$ are co-prime polynomials of $\lambda$ with real coefficients. Without any loss of generality, $H_{\mathrm{D}}(\lambda)$ and $H_{\mathrm{N}}(\lambda)$ can be described as

$$
H_{\mathrm{D}}(\lambda)=a_{0}+a_{1} \lambda+\cdots+a_{\mathrm{n}} \lambda^{\mathrm{n}}, a_{\mathrm{n}} \neq 0,
$$

X.-G. Li, J.-X. Chen, and T. Chai are with State Key Laboratory of Synthetical Automation for Process Industries (Northeastern University), Shenyang, Liaoning 110819, China. e-mail: masdanlee@163.com (X.-G. Li), chenjunxiu1024@163.com (J.-X. Chen), tychai@mail.neu.edu.cn (T. Chai).

X.-G. Li and J.-X. Chen are also with School of Information Science and Engineering, Northeastern University, Shenyang, Liaoning 110819, China.

S.-I. Niculescu is with Laboratoire des Signaux et Systèmes (L2S, UMR CNRS 8506), CNRS-CentraleSupélecUniversité Paris-Sud, Gif-Sur-Yvette 91192, France. e-mail: Silviu.Niculescuel2s. centralesupelec.fr

$$
H_{\mathrm{N}}(\lambda)=b_{0}+b_{1} \lambda+\cdots+b_{\mathrm{m}} \lambda^{\mathrm{m}}, b_{\mathrm{m}} \neq 0,
$$

with $\mathrm{n} \geq \mathrm{m}$. In practice, the control loop is subject to a delay $\tau$, and the actual transfer function is expressed as $H_{0}(\lambda) e^{-\tau \lambda}$.

In this paper, we employ the PID controller of the form:

$$
k_{P}+\frac{k_{I}}{\lambda}+k_{D} \lambda
$$

where the controller gains $k_{P}, k_{I}$, and $k_{D}$ are real parameters.

Our objective is to design PID controllers and to explicitly compute the corresponding exhaustive stability range of $\tau$ of the closed-loop system. Towards this end, we treat all the controller gains and the delay as free parameters simultaneously. To the best of the authors' knowledge, although such a problem involving totally four free parameters $\left(k_{P}, k_{I}, k_{D}\right.$, and $\tau$ ) has been largely treated, it has not received a complete characterization. One of the technical difficulties is related to the appearance of multiple and/or degenerate critical imaginary roots (CIRs) depending on the system parameters.

In the sequel, we briefly review some existing results concerning the PID stabilization for time-delay systems. A large body of results have been reported in the case of a fixed $\tau$, see e.g., [8], [23], [27], and [29]. Such a problem falls in the so-called $D$-decomposition problem [24]. By using the methods reported in the above references, all the stabilizing set for $\left(k_{P}, k_{I}, k_{D}\right)$ can be obtained. Next, the pole assignment is investigated in [31], where the dominant poles with a fixed $\tau$ may be assigned to some desired positions through adjusting the PID controller parameters. For generic systems with fixed delays, the continuous pole placement is presented in [19] and the spectral abscissa optimization is discussed in [18] and [21] (see also Chapter 7 of [20]).

However, the aforementioned results can not be applied when the delay $\tau$ is also a free parameter. Next, we recall some stabilization results for such a case. In the literature, a stability interval of $\tau$ in the form

$$
\tau \in[0, \bar{\tau}),
$$

is widely studied (stability robustness w.r.t. "small" delays), and the corresponding $\bar{\tau}$ is called the delay margin, see e.g., [7] and [20]. In [10] and [22], the upper bound for $\bar{\tau}$ is estimated. While in [28], the lower bound for $\bar{\tau}$ is estimated. The lower bound is usually more useful for practical applications: For a lower bound $\tau_{L}$, there exists an LTI controller stabilizing the plant for $\tau \in\left[0, \tau_{L}\right)$.

Although the aforementioned results are very insightful, some limitations exist: (i) The stabilization conditions are sufficient but not necessary; (ii) It is not easy to find the corresponding controllers, with which the upper/lower stabilizable 
bounds may be approached; (iii) The required controllers are often complex (two such controllers can be seen in Example 8); (iv) The stabilization set for $\tau$ is restricted to the form (3). In this paper, we will show that a plant may be stabilized with more than one interval including or excluding 0 .

The delay-independent stabilization problem is investigated in e.g., [1], [6], [9], and [25]. As the main technical issue is to ensure that there is no CIR for any $\tau$, the delay $\tau$ is not treated as a free parameter.

As far as we know, the stabilization problem addressed in this paper has not been fully investigated (though the existing algorithms cover some case studies). In this paper, we will develop a new methodology to characterize the stability set in the $\left(k_{P}, k_{I}, k_{D}, \tau\right)$-space. No constraint on the controlled plant (1) is imposed and arbitrarily complex asymptotic behavior of the CIRs is allowed. Our development consists of two stages.

(i) The first stage is to study the complete stability problem w.r.t. $\tau$ under given $\left(k_{P}, k_{I}, k_{D}\right)$. We will begin with the asymptotic behavior analysis through addressing the positive real roots of the auxiliary characteristic equation (termed the effective $W$ roots in this paper). Then, some algebraic results will be derived such that the distribution of $N U(\tau)$ can be precisely determined. Based on the $N U(\tau)$ distribution, we will develop a computationally efficient algorithm, by which the exhaustive stability range of $\tau$ can be obtained automatically. With the results derived at this stage, the stability problem in the 4-D $\left(k_{P}, k_{I}, k_{D}, \tau\right)$-space can be transformed to the complete stability problem in the 3-D $\left(k_{P}, k_{I}, k_{D}\right)$-space.

(ii) In the second stage, we will propose an approach to obtain the complete effective $W$ root classification in the whole $\left(k_{P}, k_{I}, k_{D}\right)$-space. This classification is essential: As $\left(k_{P}, k_{I}, k_{D}\right)$ continuously changes, the structure of $N U(\tau)$ expression varies iff the effective $W$ root classification varies. With the complete effective $W$ root classification, we may appropriately divide the $\left(k_{P}, k_{I}, k_{D}\right)$-space and the boundaries of different regions can be analytically determined. It should be pointed out that a full stability set characterization cannot be done without the results at this stage. As explained in the paper, the $N U(\tau)$ distribution undergoes an abrupt change at the boundaries of different regions (divided according to the complete effective $W$ root classification). Such boundaries are strongly connected to multiple effective $W$ roots and their detection is not straightforward. Therefore, a dedicated "global" study of the parameter space is necessary.

Based on the above results, we will establish a parameter-space approach to determine the stability set in $\left(k_{P}, k_{I}, k_{D}, \tau\right)$-space. Consequently, we can design the PID controller with the entire stability $\tau$-set accurately computed.

This paper is organized as follows. In Section II, some preliminaries and prerequisites are given. An algebraic algorithm for obtaining the whole stability range of $\tau$, under fixed controller gains, is proposed in Section III. In Section IV, the complete effective $W$ root classification is investigated. Some simplifications for the complete stability analysis and the complete effective $W$ root classification are proposed in Section V. The parameter-space approach for determining the stability set in the $\left(k_{P}, k_{I}, k_{D}, \tau\right)$-space is established in Section VI. Illustrative examples are provided in Section VII.
Finally, the paper concludes in Section VIII.

Notations: In this paper, $\mathbb{R}\left(\mathbb{R}_{+}\right)$denotes the set of (positive) real numbers and $\mathbb{C}$ is the set of complex numbers. For $\lambda \in \mathbb{C}$, $\operatorname{Re}(\lambda)$ and $\operatorname{Im}(\lambda)$ denote the real part and the imaginary part of $\lambda$, respectively. $\mathbb{C}_{-}\left(\mathbb{C}_{+}\right)$denotes the left-half (right-half) plane; $\mathbb{C}_{0}$ is the imaginary axis; $\mathbb{N}$ is the set of non-negative integers. $\varepsilon$ is a sufficiently small positive real number. For $\gamma \in \mathbb{R},\lceil\gamma\rceil$ denotes the smallest integer greater than or equal to $\gamma$. Finally, $\operatorname{deg}(\cdot)$ denotes the degree of a polynomial.

\section{Preliminaries and Prerequisites}

When no confusion occurs, we let $k_{I} \neq 0$ in the context of PID controller (2). If $k_{I}=0$, the PID controller (2) reduces to a PD controller (provided that $k_{P} \neq 0$ and $k_{D} \neq 0$ ).

For the closed-loop system of plant (1) subject to PID controller (2), the characteristic function is given by

$$
f(\lambda, \tau)=H_{\mathrm{D}}(\lambda) \lambda+H_{\mathrm{N}}(\lambda)\left(k_{I}+k_{P} \lambda+k_{D} \lambda^{2}\right) e^{-\tau \lambda} .
$$

The closed-loop system is asymptotically stable iff all the characteristic roots lie in $\mathbb{C}_{-}$.

We will not specifically consider a trivial case that $\lambda=0$ is a characteristic root (this property holds for all $\tau \geq 0$ ). In this case $\left(b_{0}=0\right)$, the closed-loop system can not be asymptotically stable for any $\tau \geq 0$.

\section{A. Some elementary principles}

For the closed-loop system associated with characteristic function (4), there are three possible types: retarded, neutral, and advanced, i.e., when $\operatorname{deg}\left(H_{\mathrm{D}}(\lambda) \lambda\right)-\operatorname{deg}\left(H_{\mathrm{N}}(\lambda)\left(k_{I}+\right.\right.$ $\left.\left.k_{P} \lambda+k_{D} \lambda^{2}\right)\right)>0,=0$, and $<0$, respectively.

For a retarded-type (advanced-type) system, as $\tau$ increases from 0 to $+\varepsilon$, an arbitrarily small positive real number, infinitely many new characteristic roots appear at the far left (right) of the complex plane.

For a neutral-type system, as $\tau$ increases from 0 to $+\varepsilon$, infinitely many new characteristic roots appear between two vertical lines in the complex plane, and hence the positions of the vertical lines lead to a necessary stability condition (the below constraints (5) and (7) are due to it). For more details, one may refer to Section 1.2 of [20] or Chapter 10 of [12].

We have three elementary principles for adopting the PID controller.

Elementary principle 1: In the case $\operatorname{deg}\left(H_{\mathrm{D}}(\lambda)\right) \geq$ $\operatorname{deg}\left(H_{\mathrm{N}}(\lambda)\right)+2$, the PID controller (2) may be applied. The closed-loop system is of the retarded type.

Elementary principle 2: In the case $\operatorname{deg}\left(H_{\mathrm{D}}(\lambda)\right)=$ $\operatorname{deg}\left(H_{\mathrm{N}}(\lambda)\right)+1$, the PID controller (2) may be applied. The closed-loop system is of the neutral type and the gain $k_{D}$ has to satisfy the condition

$$
\left|k_{D}\right|<\left|\frac{a_{n}}{b_{m}}\right| .
$$

If $k_{D}=0$, the PID controller reduces to a PI controller

$$
k_{P}+\frac{k_{I}}{\lambda} \text {. }
$$

The PI controller (6) may be used, under which the closedloop system is of the retarded type. 
Elementary principle 3: In the case $\operatorname{deg}\left(H_{\mathrm{D}}(\lambda)\right)=$ $\operatorname{deg}\left(H_{\mathrm{N}}(\lambda)\right)$, the PID controller (2) with $k_{D} \neq 0$ can not stabilize the plant. The PI controller (6) may be used. The closed-loop system is of the neutral type and the gain $k_{P}$ has to satisfy the condition

$$
\left|k_{P}\right|<\left|\frac{a_{n}}{b_{m}}\right| .
$$

\section{B. Complete stability problem w.r.t. delay parameter}

For a given controller vector $\left(k_{P}, k_{I}, k_{D}\right)$, the complete stability problem w.r.t. $\tau$ refers to the problem of studying the stability property along the whole non-negative $\tau$-axis.

By the root continuity argument, $N U(\tau)$ changes as $\tau$ increases only when the system has a critical imaginary root (CIR) $\lambda=j \omega, \omega \in \mathbb{R}$, at some $\tau$. These delays are called the critical delays (CDs). A pair $(\lambda, \tau)$, where $\lambda \in \mathbb{C}_{0}$ and $\tau \in \mathbb{R}_{+} \cup\{0\}$, such that $f(\lambda, \tau)=0$ is called a critical pair.

The generic solution for the complete stability problem consists of two tasks: First, an exhaustive detection of the CIRs and the CDs. Second, the asymptotic behavior analysis of the CIRs w.r.t. the infinitely many CDs. Due to the conjugate symmetry of the spectrum, it suffices to consider only the CIRs with non-negative imaginary parts.

Without any loss of generality, suppose that there exist $u \in$ $\mathbb{N}$ CIRs, denoted by $\lambda_{\alpha}=j \omega_{\alpha}, \alpha=0, \ldots, u-1$. For a CIR $\lambda_{\alpha}=j \omega_{\alpha}$, the infinitely many CDs are denoted by $\tau_{\alpha, k}=$ $\tau_{\alpha, 0}+\frac{2 k \pi}{\omega_{\alpha}}, k \in \mathbb{N}$, where $\tau_{\alpha, 0}$ is the minimum non-negative value of $\tau$ satisfying the condition

$$
e^{-\tau \omega_{\alpha} j}=-\frac{H_{\mathrm{D}}\left(j \omega_{\alpha}\right) j \omega_{\alpha}}{H_{\mathrm{N}}\left(j \omega_{\alpha}\right)\left(k_{I}+k_{P} j \omega_{\alpha}-k_{D} \omega_{\alpha}^{2}\right)} .
$$

Property 1: For the closed-loop system described by (1) and (2), a non-zero critical imaginary root only corresponds to one set of critical delays.

Property 1 may be proved in the line of Property 1 of [14].

For a critical pair $\left(\lambda_{\alpha}, \tau_{\alpha, k}>0\right)$, the effect of its asymptotic behavior on $N U(\tau)$, can be quantified by means of the notation $\Delta N U_{\lambda_{\alpha}}\left(\tau_{\alpha, k}\right) \in \mathbb{N}$. As defined in [12], $\Delta N U_{\lambda_{\alpha}}\left(\tau_{\alpha, k}\right)$ stands for the change of $N U(\tau)$ caused by the variation of the CIR $\lambda_{\alpha}$ as $\tau$ increases from $\tau_{\alpha, k}-\varepsilon$ to $\tau_{\alpha, k}+\varepsilon$.

\section{Algorithm for Complete Stability Analysis UNDER FiXed CONTROLler PARAMETERS}

The objective of this section is to develop some algebraic criteria for the complete stability analysis under any given $\left(k_{P}, k_{I}, k_{D}\right)$. An algorithm such that the entire stability range of $\tau$ can be automatically computed will also be presented.

\section{A. Auxiliary characteristic function}

Following the line of Section 2 in [5], we can straightforwardly have the following lemma.

Lemma 1: For given $k_{P}, k_{I}$, and $k_{D}, \lambda=j \omega \neq 0$ is a critical imaginary root for the closed-loop system described by (1) and (2) iff $W=\omega^{2}$ is a positive real root for the auxiliary characteristic equation $F_{\mathrm{PID}}(W)=0$, where $F_{\mathrm{PID}}(W)$ is the auxiliary characteristic function given by

$$
\begin{aligned}
& F_{\mathrm{PID}}(W)=\operatorname{Re}\left(H_{\mathrm{D}}(j \omega) j \omega\right)^{2}+\operatorname{Im}\left(H_{\mathrm{D}}(j \omega) j \omega\right)^{2} \\
& -\operatorname{Re}\left(H_{\mathrm{N}}(j \omega)\left(k_{I}+k_{P} j \omega-k_{D} \omega^{2}\right)\right)^{2} \\
& -\operatorname{Im}\left(H_{\mathrm{N}}(j \omega)\left(k_{I}+k_{P} j \omega-k_{D} \omega^{2}\right)\right)^{2} .
\end{aligned}
$$

The right-hand side of (9) is a polynomial of $\omega$ with only even orders, and hence the auxiliary characteristic function may be denoted as a polynomial of $W=\omega^{2}$.

As we have precluded the case $\lambda=0$ is characteristic root, we will not consider the case $F_{\mathrm{PID}}(0)=0$. Since $W=\omega^{2}$, we are only interested in the positive real roots, called the effective $W$ roots. Consistent with the CIRs, there are $u$ effective $W$ roots denoted by $W_{\alpha}, \alpha=0, \ldots, u-1$, with $W_{\alpha}=\omega_{\alpha}^{2}$.

The application of auxiliary characteristic function may be traced back to [5]. However, the existing methods have strong restrictions. It is pointed out in [32] that it is an open issue to address the multiple auxiliary characteristic roots. In the sequel, we will give some new results covering the open issue.

\section{B. Asymptotic behavior and auxiliary characteristic roots}

Without any loss of generality, suppose that among the effective $W$ roots there are $q_{o} \in \mathbb{N}\left(q_{e} \in \mathbb{N}\right)$ ones with odd (even) multiplicities, denoted by $W_{0}^{o}, \ldots, W_{q_{o}-1}^{o}$ $\left(W_{0}^{e}, \ldots, W_{q_{e}-1}^{e}\right)$. We arrange them as:

$$
W_{0}^{o}>\cdots>W_{q_{o}-1}^{o}>0, W_{0}^{e}>\cdots>W_{q_{e}-1}^{e}>0 .
$$

For each $W_{i}^{\dagger}$ († is " $o$ " or " $e$ "), we denote the corresponding critical pairs by $\left(\lambda_{i}^{\dagger}=j \omega_{i}^{\dagger}, \tau_{i, k}^{\dagger}\right)$, where $\omega_{i}^{\dagger}=\sqrt{W_{i}^{\dagger}}>0$ and $\tau_{i, k}^{\dagger}=\tau_{i, 0}^{\dagger}+\frac{2 k \pi}{\omega_{i}^{\dagger}}(k \in \mathbb{N})$ are the associated CDs. In light of Theorem 2 in [14], we have the following algebraic criterion:

Theorem 1: For the closed-loop system described by (1) and (2) with given gains $k_{P}, k_{I}$, and $k_{D}$, the following two statements hold:

(1) For a critical imaginary root $\lambda_{i}^{e}=j \omega_{i}^{e}, \Delta N U_{\lambda_{i}^{e}}\left(\tau_{i, k}^{e}\right)=$ 0 for all $\tau_{i, k}^{e}>0(k \in \mathbb{N})$.

(2) For a critical imaginary root $\lambda_{i}^{o}=j \omega_{i}^{o}, \Delta N U_{\lambda_{i}^{o}}\left(\tau_{i, k}^{o}\right)=$ $+1(-1)$ for all $\tau_{i, k}^{o}>0(k \in \mathbb{N})$ if $i$ is even (odd).

Next, based on Theorem 1, we can easily derive the expression of $N U(\tau)$ according to the root continuity argument.

Theorem 2: Consider the closed-loop system described by (1) and (2) with given $k_{P}, k_{I}$, and $k_{D}$. For any $\tau>0$ which is not a critical delay, $N U(\tau)$ can be explicitly expressed as

$$
N U(\tau)=N U(+\varepsilon)+\sum_{i=0}^{q_{o}-1} N U_{i}^{o}(\tau),
$$

where

$$
\begin{aligned}
& N U_{i}^{o}(\tau)=\left\{\begin{array}{l}
0, \tau<\tau_{i, 0}^{o}, \\
(-1)^{i} 2\left\lceil\frac{\tau-\tau_{i, 0}^{o}}{2 \pi / \omega_{i}^{o}}\right], \tau>\tau_{i, 0}^{o},
\end{array}, \text { if } \tau_{i, 0}^{o} \neq 0,\right. \\
& N U_{i}^{o}(\tau)=\left\{\begin{array}{l}
0, \tau<\tau_{i, 1}^{o}, \\
(-1)^{i} 2\left\lceil\frac{\tau-\tau_{i, 1}^{o}}{2 \pi / \omega_{i}^{o}}\right], \tau>\tau_{i, 1}^{o},
\end{array} \text { if } \tau_{i, 0}^{o}=0 .\right.
\end{aligned}
$$

(The value of $N U(+\varepsilon)$ can be calculated according to Theorem 5.1 in [12] or Theorem 1 in [13]) 
Now we can obtain the $N U(\tau)$ distribution w.r.t. $\tau$ along the whole $\tau$-axis. Consequently, we may determine the whole stability range of $\tau$ through addressing the $N U(\tau)$ distribution.

Remark 1: The structure of $N U(\tau)$ is determined by the number of effective $W$ roots and their respective multiplicities, in light of Theorems 1 and 2. This observation will play an important role in our global study of the parametric space.

In the next subsection, we will develop an algorithm by which all the stability range of $\tau$ can be automatically detected for a fixed $\left(k_{P}, k_{I}, k_{D}\right)$.

\section{Algebraic algorithm for complete stability analysis}

Corollary 1: The closed-loop system described by (1) and (2) must belong to the following three cases:

(1) Asymptotically stable for all $\tau \in[0, \infty)$.

(2) Asymptotically stable along the whole $\tau$-axis except at some isolated points.

(3) There exists a $\tau^{*} \geq 0$ such that $N U(\tau)>0$ for all $\tau \geq \tau^{*}$.

Case (1) happens iff $N U(0)=0$ and there is no effective $W$ root. Case (2) happens iff $N U(+\varepsilon)=0$ and there are only effective $W$ roots with even multiplicities. In the other situations, Case (3) occurs.

One may easily prove the above corollary by combining the idea of Theorem 9.2 in [12] (or Theorem 7 in [13]) and the algebraic results introduced previously in this section.

Based on Corollary 1, we will derive a computationally efficient algorithm for the complete stability analysis. Our idea is: Cases (1) and (2) can be easily determined according to the effective $W$ roots and $N U(+\varepsilon)$. If these two cases are excluded, i.e., in Case (3), we may choose a sufficiently large $\tau^{*}$ and keep track of the finite-length $N U(\tau)$ distribution for $0 \leq \tau \leq \tau^{*}$. In this way, the exhaustive stability range of $\tau$ may be detected (details will be given in Algorithm 1).

When $\tau=0$, the characteristic function (4) reduces to

$$
f(\lambda, 0)=H_{\mathrm{D}}(\lambda) \lambda+H_{\mathrm{N}}(\lambda)\left(k_{I}+k_{P} \lambda+k_{D} \lambda^{2}\right) .
$$

All the $n+1$ characteristic roots can be computed by a computer, since (12) is an $(n+1)$ th-order polynomial.

We define by $\mathcal{N}_{W+}^{\tau=0}$ the set in the $\left(k_{P}, k_{I}, k_{D}\right)$-space where there is at least one effective $W$ root when $\tau=0$ (i.e., the closed-loop system has CIRs when $\tau=0$ ).

For a $\left(k_{P}, k_{I}, k_{D}\right) \notin \mathcal{N}_{W+}^{\tau=0}$, it is obvious that $N U(+\varepsilon)=$ $N U(0)$. We now present the algorithm for the complete stability analysis for a $\left(k_{P}, k_{I}, k_{D}\right) \notin \mathcal{N}_{W+}^{\tau=0}$ (the case of $\left(k_{P}, k_{I}, k_{D}\right) \in \mathcal{N}_{W+}^{\tau=0}$ will be discussed in Subsection VI-A).

Algorithm 1 (complete stability analysis w.r.t. $\tau$ ):

Step 1: Determine $N U(0)$ through solving the equation $f(\lambda, 0)=0$, where $f(\lambda, 0)$ is given in (12).

Step 2: Solve the auxiliary characteristic equation $F_{\text {PID }}(W)=0$. If there is no effective $W$ root, go to Step 3 . If there are only effective $W$ roots with even multiplicities, go to Step 4. Otherwise, go to Step 5.

Step 3: The closed-loop system is asymptotically stable (unstable) for all $\tau \in[0, \infty)$ if $N U(0)=0(>0)$. Go to Step 6.

Step 4: The closed-loop system is asymptotically stable along the whole $\tau$-axis except at the CDs if $N U(0)=0$.
The closed-loop system can not be asymptotically stable at any $\tau$ if $N U(0)>0$. Go to Step 6 .

Step 5: Choose a sufficiently large $\tau^{*}>0$. Obtain all the critical pairs $(\lambda, \tau)$ where the CDs are not larger than $\tau^{*}$. These CDs divide $\left[0, \tau^{*}\right]$ into finitely many open intervals. At all these CDs, the corresponding values of $\Delta N U_{\lambda}(\tau)$ can be computed by Theorem 1. Then, using Theorem 2, we can determine the value of $N U(\tau)$ for each open interval. An open interval of $\tau$ with $N U(\tau)=0$ is a stability interval. As a result, all the stability intervals of $\tau$ (if any) may be explicitly computed.

Step 6: The algorithm stops.

For the complete stability problem of time-delay systems, various approaches have been proposed, see e.g., [5], [13], [26], and [30]. However, most of the existing results have technical limitations. Algorithm 1 covers the general case for the PID stabilization problem of time-delay systems (this will be demonstrated by various examples in Section VII) and, moreover, it will be used in the sequel for the scenario where the controller gains are also free parameters.

\section{Complete efFective $W$ ROOT Classification}

By using the results of the last section, for any PID controller vector $\left(k_{P}, k_{I}, k_{D}\right)$, the $N U(\tau)$ distribution can be algebraically determined. Along this line, the stability analysis over the 4-D $\left(k_{P}, k_{I}, k_{D}, \tau\right)$-space may be recast into the complete stability analysis over the 3 -D $\left(k_{P}, k_{I}, k_{D}\right)$-space.

Our next task is to have some global characterization concerning the $N U(\tau)$ distribution over the $\left(k_{P}, k_{I}, k_{D}\right)$ space. Remark 1 provides a path along analyzing how the number of effective $W$ roots and their multiplicities change over the whole $\left(k_{P}, k_{I}, k_{D}\right)$-space. Such an analysis is called the complete effective $W$ root classification.

The contribution of this section is two-fold: (1) The whole parameter space of $\left(k_{P}, k_{I}, k_{D}\right)$ will be properly divided into some regions. In each region, the $N U(\tau)$ expression has a fixed structure. (2) The boundaries of different regions divided in the $\left(k_{P}, k_{I}, k_{D}\right)$-space, where the $N U(\tau)$ distribution undergoes an abrupt change, may be analytically determined.

We will develop an approach for the complete effective $W$ root classification, by taking into account the characteristics of closed-loop system with PID controller. We first review a mathematical tool for polynomial algebra.

\section{A. Discrimination system}

For a real-coefficient polynomial

$$
Q(y)=c_{q} y^{q}+c_{q-1} y^{q-1}+\cdots+c_{0}, c_{q} \neq 0,
$$

the root classification refers to the information concerning the numbers and multiplicities of the distinct real and complex roots of $Q(y)=0$.

The complete root classification of $Q(y)$ is the collection of its all possible root classifications, together with the conditions on the parametric coefficients such that each root classification is realized. The complete root classification can be systematically solved by a generic tool, called the discrimination system (see [33] and the references therein). 
For the $q$ th-order polynomial $Q(y)$ (13), the discrimination matrix is the following $2 q \times 2 q$ matrix $M=$

$$
\left(\begin{array}{cccccccc}
c_{q} & c_{q-1} & c_{q-2} & \ldots & c_{0} & & & \\
0 & q c_{q} & (q-1) c_{q-1} & \ldots & c_{1} & & \\
& c_{q} & c_{q-1} & \ldots & c_{1} & c_{0} & & \\
& 0 & q c_{q} & \ldots & 2 c_{2} & c_{1} & & \\
& & & \vdots & \vdots & & & \\
& & & c_{q} & c_{q-1} & c_{q-2} & \ldots & c_{0} \\
& & & 0 & q c_{q} & (q-1) c_{q-1} & \ldots & c_{1}
\end{array}\right)
$$

Let $D_{\alpha}$ denote the determinant of the submatrix of $M$ formed by the first $2 \alpha$ rows and the first $2 \alpha$ columns for $\alpha=1, \ldots, q$. The $q$-tuple $D=\left[D_{1}, \ldots, D_{q}\right]$ is called the discriminant sequence of $Q(y)$. According to the signs of $D_{1}, \ldots, D_{q}$, we have the revised sign list for $Q(y)$.

Proposition 1 ([33]): If the revised sign list for $Q(y)$ has $l$ non-vanishing elements and the number of sign changes is $v$, then $Q(y)$ has $v$ pairs of distinct complex conjugate roots and $l-2 v$ distinct real roots.

If Proposition 1 is not sufficient for concluding on the complete root classification (more precisely, it may be insufficient to determine the multiplicity information), we construct the so-called $\Delta$-sequence of $Q(y)$. Then, through analyzing the complete root classification of the $\Delta$-sequence (by using Proposition 1), we may determine the complete root classification of $Q(y)$ (i.e., the multiplicity information can also be available). For more details, please refer to [33].

To summarize, for the polynomial $Q(y)$ (13), we may obtain a set of explicit expressions in terms of the coefficients, called the discrimination system, which determines all the possible cases on the numbers and multiplicities of the real roots.

\section{B. Complete effective $W$ root classification}

As the auxiliary characteristic function $F_{\mathrm{PID}}(W)$ is an $(n+1)$ th-order polynomial, the discriminant sequence is of the form

$$
\left[D_{1}, \ldots, D_{n+1}\right] .
$$

Applying the discrimination system, we can classify the distribution of real $W$ roots in the $\left(k_{P}, k_{I}, k_{D}\right)$-space, i.e., obtain the complete real $W$ root classification, and divide the whole $\left(k_{P}, k_{I}, k_{D}\right)$-space into regions accordingly. We denote them by $\mathcal{N}_{W}^{\{,, \ldots, \cdot\}}$. For instance: " $\mathcal{N}_{W}^{\{1,1,1\} \text { " denotes a region }}$ with three distinct simple real $W$ roots; " $\mathcal{N}_{W}^{\{2,1\} \text { " denotes a }}$ region with one double real $W$ root plus one simple real $W$ root; " $\mathcal{N}_{W}^{\{\}}$" denotes a region without a real $W$ root.

Among the real $W$ roots, we need to filter out the negative ones (since $W=\omega^{2}$ ). To this end, in this subsection we will give a novel approach to obtain the complete effective $W$ root classification, i.e., the complete positive real $W$ root classification, in the $\left(k_{P}, k_{I}, k_{D}\right)$-space.

We start by examining where a real $W$ root may change its sign (i.e., a negative real $W$ root becomes a positive real $W$ root or the other way) in the $\left(k_{P}, k_{I}, k_{D}\right)$-space. There must exist a critical state where $W=0$ is an auxiliary characteristic root if a real $W$ root changes its sign.

In view of (9), the following property is true.
Property 2: For the closed-loop system described by (1) and (2), there exists an auxiliary characteristic root $W \rightarrow 0$ iff $k_{I} \rightarrow 0$.

We have the main theorem of this subsection:

Theorem 3: For the closed-loop system described by (1) and (2), consider a region divided according to the complete real $W$ root classification. If this region is separated by the set $k_{I}=0$ in the $\left(k_{P}, k_{I}, k_{D}\right)$-space, then the effective $W$ root classification does not change in each subregion of this region separated by the set $k_{I}=0$. Otherwise, the effective $W$ root classification does not change in the whole region.

Proof: If a $\left(k_{P}, k_{I}, k_{D}\right)$-point continuously changes in a region divided according to the complete real $W$ root classification, all the real $W$ roots keep their signs if this point does not intersect the set $k_{I}=0$, by Property 2 .

The set $k_{I}=0$ is nothing but a line in the 2-D parameter space or a plane in the 3-D parameter space. In light of Theorem 3, we have a computationally efficient procedure for the complete effective $W$ root classification.

\section{Procedure 1 (complete effective $W$ root classification):}

Step 1: Apply the discrimination system to the auxiliary characteristic function $F_{\mathrm{PID}}(W)$. The complete real $W$ root classification in the whole $\left(k_{P}, k_{I}, k_{D}\right)$-space can be obtained.

Step 2: For each region divided according to the complete real $W$ root classification, we determine all possible effective $W$ root classifications in light of Theorem 3. More precisely, for each region not separated by the set $k_{I}=0$ (subregion separated by the set $k_{I}=0$ ), we choose any point to solve the auxiliary characteristic equation $F_{\mathrm{PID}}(W)=0$ and the effective $W$ root classification at this point is exactly the same as for all the points in the region (subregion).

Moreover, the analytic boundaries of different effective $W$ root classifications in the $\left(k_{P}, k_{I}, k_{D}\right)$-space are available and can be explicitly computed (see the analysis in detail in Subsection IV-C).

Remark 2: An alternative way for the complete effective $W$ root classification is: Let $W=V^{2}$ and then analyze the complete real $V$ root classification for $F_{\mathrm{PID}}\left(V^{2}\right)=0$. However, the computational complexity for employing the discrimination system substantially increases. Procedure 1 combines the discrimination system and the characteristics of the closed-loop system with PID controller. This procedure considerably reduces the computational complexity.

In the sequel, we introduce underlines and overlines to the notation $\mathcal{N}_{W}^{\{, \ldots, \cdot\}}$ to clarify the sign of each real $W$ root. Furthermore, we denote the regions divided according to the complete effective $W$ root classification by $\mathcal{N}_{W_{+}}^{\{\cdot, \ldots, \cdot\}}$. For instance: If a region $\mathcal{N}_{W}^{\{1,1,1\}}$ (with three distinct simple real $W$ roots) is with two simple negative real $W$ roots and one simple positive real $W$ root, we denote this region by

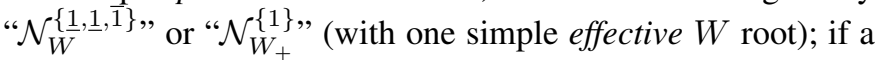
region $\mathcal{N}_{W}^{\{2,1\}}$ (with one double real $W$ root plus one simple real $W$ root) is with one double positive real $W$ root and one simple negative real $W$ root, we denote this region by

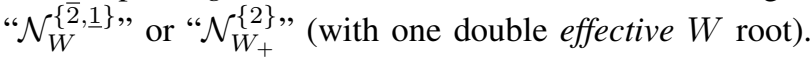

The above description may by further simplified by introducing an arrow " $\rightarrow$ ". For instance, we may simply use 


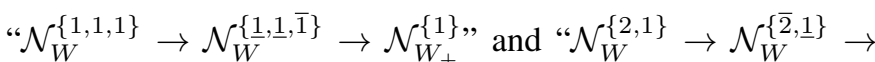

$\mathcal{N}_{W_{+}}^{\{2\}}$, to summarize the two cases in the last paragraph.

\section{Boundaries of different effective $W$ root classifications}

We first give a useful property.

Property 3: For the closed-loop system described by (1) and (2), as $\left(k_{P}, k_{I}, k_{D}\right)$ continuously varies, the effective $W$ root classification changes iff the $\left(k_{P}, k_{I}, k_{D}\right)$-point intersects a region with multiple effective $W$ roots or the plane $k_{I}=0$.

Proof: The characteristic function (4) is with real coefficients, and hence all the complex $W$ roots appear in complex conjugate pairs. As $\left(k_{P}, k_{I}, k_{D}\right)$ continuously varies, if the effective $W$ root classification changes, the following cases are possible.

Case (1): The total number of effective $W$ roots (multiplicity taken into account) changes. More precisely, Case (1) has two sub-cases. Sub-case (1.1): A pair of complex conjugate $W$ roots collide on the real axis (a double effective $W$ root appears and the total number of effective $W$ roots increases by two) or a double effective $W$ root splits into two complex conjugate $W$ roots (a double effective $W$ root disappears and the total number of effective $W$ roots decreases by two). Subcase (1.2): A positive real $W$ root becomes a $W=0$ root (total number of effective $W$ roots decreases by one) or a $W=0$ root becomes a positive real $W$ root (total number of effective $W$ roots increases by one).

Case (2): The total number of effective $W$ roots (multiplicity taken into account) does not change. However, the multiplicity information changes. Case (2) happens if real $W$ roots collide or split along the real axis.

Sub-case (1.1) and Case (2) happen on the set with multiple effective $W$ roots. Sub-case (1.2) occurs on the set $k_{I}=0$, according to Property 2.

We next focus on the regions with multiple effective $W$ roots.

Property 4: For the auxiliary characteristic equation $F_{\text {PID }}(W)=0$, there are multiple effective $W$ roots only if $D_{n+1}=0$.

It is a generic property related with the discrimination system that there is a multiple (real or complex) root iff the last element of the discrimination sequence equals to 0 (one may prove it by contradiction). As $D_{n+1}$ is a polynomial of $k_{P}, k_{I}$, and $k_{D}$, a region satisfying $D_{n+1}=0$ is in general with measure 0 in the $\left(k_{P}, k_{I}, k_{D}\right)$-space.

We refer to a region at which the effective $W$ root classification changes as a boundary of different effective $W$ root classifications, because such a region can be interpreted as a boundary surface (transition) of different effective $W$ root classifications, in light of the above analysis. We can obtain the analytic conditions of a boundary from the complete effective $W$ classification and hence we are able to accurately determine a boundary, although with measure 0 .

Remark 3: It is seen from the proof of Property 3 that for almost all closed-loop systems with PID controllers, there exist sets of $\left(k_{P}, k_{I}, k_{D}\right)$ parameters that result in multiple real $W$ roots. Since all the complex $W$ roots appear in complex conjugate pairs, the collision (if any) of a pair of complex conjugate $W$ roots, as $\left(k_{P}, k_{I}, k_{D}\right)$ continuously changes, must occur on the real axis. This gives rise to at least a double real $W$ root.

\section{Effective $W$ root classification and $N U(\tau)$ distribution}

Now, we know that the $N U(\tau)$ distribution may have two types of variations:

(1) In a region divided according to the complete effective $W$ root classification, the $N U(\tau)$ distribution continuously varies w.r.t. $\left(k_{P}, k_{I}, k_{D}\right)$.

(2) On the boundaries where the effective $W$ root classification changes, the $N U(\tau)$ distribution has an abrupt change w.r.t. $\left(k_{P}, k_{I}, k_{D}\right)$.

Remark 4: Owing to the complete effective $W$ root classification proposed in this paper, even if the $N U(\tau)$ distribution has an abrupt change, we are able to accurately detect it. Moreover, inside a region with the same effective $W$ root classification, we may apply the parameter-sweeping technique to study the $N U(\tau)$ distribution. It is worth mentioning that such a parameter sweeping is for the quantitative test.

Based on the results above, we will propose a systematic approach in Section VI to examine the stability set in the space of $\left(k_{P}, k_{I}, k_{D}, \tau\right)$. With the complete effective $W$ root classification, the solution will be complete from the theoretical as well as the computational viewpoint.

\section{E. Illustrative explanatory examples}

Example 1: Consider the controlled plant (40) of [28]

$$
H_{0}(\lambda)=\frac{1}{(\lambda-0.2)(\lambda-1)} .
$$

Following the discussions in Subsection II-A, we employ the PID controller to this plant. For a clear illustration, we here analyze the case $k_{P}=-0.1$ and $\left(k_{I}, k_{D}\right) \in[0.01,0.2] \times$ $[0.01,1.7]$. We now apply Procedure 1 .

The auxiliary characteristic function is $F_{\mathrm{PID}}(W)=W^{3}+$ $\left(-k_{D}^{2}+\frac{26}{25}\right) W^{2}+\left(-k_{P}^{2}+2 k_{I} k_{D}+\frac{1}{25}\right) W-k_{I}^{2}$.

Step 1: The discrimination sequence of $F_{\mathrm{PID}}(W)$ is $\left[D_{1}, D_{2}, D_{3}\right]$, where $D_{1}=3, D_{2}=2 k_{D}^{4}-\frac{104 k_{D}^{2}}{25}-12 k_{I} k_{D}+$ $6 k_{P}^{2}+\frac{1202}{625}, D_{3}=-4 k_{D}^{5} k_{I} k_{P}^{2}+\frac{4 k_{D}^{5} k_{I}}{25}+\frac{104 k_{D}^{4} k_{I}^{2}}{25}+k_{D}^{4} k_{P}^{4}-$ $\frac{2 k_{D}^{4} k_{P}^{2}}{25}+\frac{k_{D}^{4}}{625}+4 k_{D}^{3} k_{I}^{3}+\frac{208 k_{D}^{3} k_{I} k_{P}^{2}}{25}-\frac{208 k_{D}^{3} k_{I}}{625}+30 k_{D}^{2} k_{I}^{2} k_{P}^{2}-$ $\frac{6158 k_{D}^{2} k_{I}^{2}}{625}-\frac{52 k_{D}^{2} k_{P}^{4}}{25}+\frac{104 k_{D}^{2} k_{P}^{2}}{625}-\frac{52 k_{D}^{2}}{15625}-\frac{936 k_{D} k_{I}^{3}}{25}-24 k_{D} k_{I} k_{P}^{4}-$ $\frac{1504 k_{D} k_{I} k_{P}^{2}}{625}+\frac{2104 k_{D} k_{I}}{15625}-27 k_{I}^{4}+\frac{468 k_{I}^{2} k_{P}^{2}}{25}+\frac{58604 k_{I}^{2}}{15625}+4 k_{P}^{6}+$ $\frac{376 k_{P}^{2}}{625}-\frac{1052 k_{P}^{2}}{15625}+\frac{57625}{390625}$.

Then, by using the discrimination system, we may divide the selected domain into the following regions (Fig. 1(a)): Region A with $\mathcal{N}_{W}^{\{1,1,1\}}: D_{2}>0 \cap D_{3}>0$; Region B with $\mathcal{N}_{W}^{\{2,1\}}$ : $D_{2}>0 \cap D_{3}=0$; Region C with $\mathcal{N}_{W}^{\{1\}}:\left(D_{2}>0 \cap D_{3}<0\right)$ $\cup\left(D_{2}=0 \cap D_{3}<0\right) \cup\left(D_{2}<0 \cap D_{3}<0\right)$; Region D with $\mathcal{N}_{W}^{\{2,1\}}: D_{2}>0 \cap D_{3}=0$; Region E with $\mathcal{N}_{W}^{\{1,1,1\}}$ : $D_{2}>0 \cap D_{3}>0$.

Step 2: For each region above we may choose any point to determine the effective $W$ root classification (the selected domain is not separated by the line $k_{I}=0$ ). In practice, we do not need to specifically address the regions with measure 0 , as the effective $W$ root classifications may be implied from the 


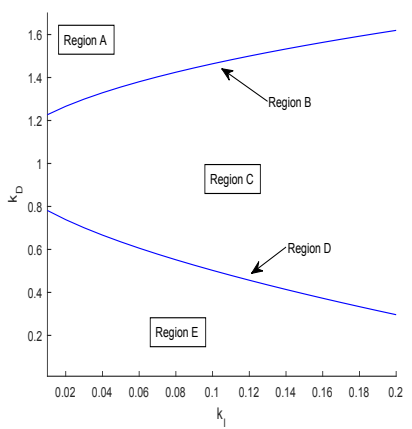

(a) Real $W$ root classifications

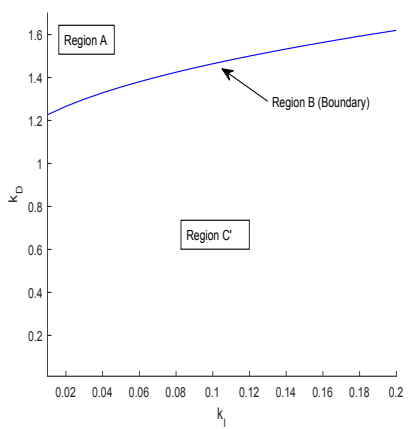

(b) Effective $W$ root classifications

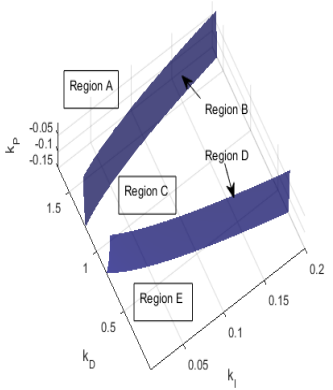

(a) Real $W$ root classifications

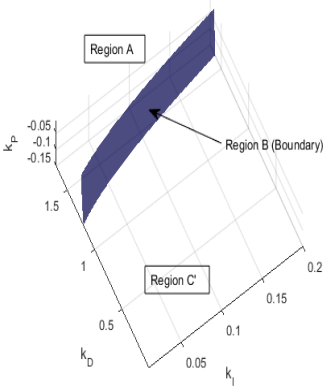

(b) Effective $W$ root classifications
Fig. 1. Complete real and effective $W$ root classifications for Example 1

TABLE I

COMPARISON OF COMPUTATIONAL COMPLEXITY

\begin{tabular}{c|c|c}
\hline & $F_{\mathrm{PID}}(W)$ & $F_{\mathrm{PID}}\left(V^{2}\right)$ \\
\hline Size of discrimination matrix & $6 \times 6$ & $12 \times 12$ \\
\hline Discrimination sequence & {$\left[D_{1}, D_{2}, D_{3}\right]$} & {$\left[D_{1}, \cdots, D_{6}\right]$} \\
\hline Maximum degree & $8\left(\right.$ degree of $\left.D_{3}\right)$ & $18\left(\right.$ degree of $\left.D_{6}\right)$ \\
\hline
\end{tabular}

ones of neighbouring regions. For this example, the effective $W$ root classifications for Region B (Region D) can be implied from the ones of Regions $\mathrm{A}$ and $\mathrm{C}$ (Regions $\mathrm{C}$ and $\mathrm{E}$ ).

According to Theorem 3, we have the complete effective $W$ root classification: Region A: $\mathcal{N}_{W}^{\{1,1,1\}} \rightarrow \mathcal{N}_{W}^{\{\overline{1}, \overline{1}, \overline{1}\}} \rightarrow$ $\mathcal{N}_{W_{+}}^{\{1,1,1\}}$; Region B: $\mathcal{N}_{W}^{\{2,1\}} \rightarrow \mathcal{N}_{W}^{\{\overline{2}, \overline{1}\}} \rightarrow \mathcal{N}_{W+}^{\{2,1\}}$; Region C: $\mathcal{N}_{W}^{\{1\}} \rightarrow \mathcal{N}_{W}^{\{\overline{1}\}} \rightarrow \mathcal{N}_{W+}^{\{1\}}$; Region D: $\mathcal{N}_{W}^{\{2,1\}} \rightarrow \mathcal{N}_{W}^{\{2, \overline{1}\}} \rightarrow$ $\mathcal{N}_{W_{+}}^{\{1\}}$; Region E: $\left.\mathcal{N}_{W}^{\{1,1,1\}} \rightarrow \mathcal{N}_{W}^{\{1,1,1}\right\} \rightarrow \mathcal{N}_{W_{+}}^{\{1\}}$.

Regions $\mathrm{C}, \mathrm{D}$, and $\mathrm{E}$ may be combined into one region, denoted by Region $\mathrm{C}^{\prime} \triangleq$ Region $\mathrm{C} \cup$ Region $\mathrm{D} \cup$ Region E. As a consequence, we may divide the selected domain into three regions (Fig. 1(b)): Region A, Region B, and Region $\mathrm{C}^{\prime}$. Region B is the boundary between Region A and Region $\mathrm{C}^{\prime}$, with the analytic condition: $D_{2}>0 \cap D_{3}=0$.

Finally, in Table I, we compare Procedure 1 with the standard method mentioned in Remark 2. It illustrates that Procedure 1 is more practical to implement.

Example 2: We now address the complete effective $W$ root classification for the system of Example 1 over the 3-D $\left(k_{P}, k_{I}, k_{D}\right)$-space. For a clear illustration, we let $\left(k_{P}, k_{I}, k_{D}\right) \in[-0.15,-0.01] \times[0.01,0.2] \times[0.01,1.7]$.

By using Procedure 1, the domain may be divided into Regions A, B, C, D, and E (Fig. 2(a)): Region A: $\mathcal{N}_{W}^{\{1,1,1\}} \rightarrow \mathcal{N}_{W}^{\{\overline{1}, \overline{1}, \overline{1}\}} \rightarrow \mathcal{N}_{W_{+}}^{\{1,1,1\}} ;$ Region B: $\mathcal{N}_{W}^{\{2,1\}} \rightarrow$ $\mathcal{N}_{W}^{\{\overline{2}, \overline{1}\}} \rightarrow \mathcal{N}_{W+}^{\{2,1\}}$; Region C: $\mathcal{N}_{W}^{\{1\}} \rightarrow \mathcal{N}_{W}^{\{\overline{1}\}} \rightarrow \mathcal{N}_{W+}^{\{1\}}$; Region D: $\mathcal{N}_{W}^{\{2,1\}} \rightarrow \mathcal{N}_{W}^{\{2, \overline{1}\}} \rightarrow \mathcal{N}_{W+}^{\{1\}}$; Region E: $\mathcal{N}_{W}^{\{1,1,1\}} \rightarrow$ $\mathcal{N}_{W}^{\{1,1, \overline{1}\}} \rightarrow \mathcal{N}_{W_{+}}^{\{1\}}$. Regions C, D, and E may be combined as Region $\mathrm{C}^{\prime} \triangleq$ Region $\mathrm{C} \cup$ Region $\mathrm{D} \cup$ Region E (Fig. 2(b)).

We next verify the above results through choosing point " 1 " (in Region $\left.\mathrm{C}^{\prime}\right)$ : $\left(k_{P}=-0.1, k_{I}=0.1, k_{D}=1.46404\right)$, point "2" (on Region B): $\left(k_{P}=-0.1, k_{I}=0.1, k_{D}=1.46405\right)$, and point "3" (in Region A): $\left(k_{P}=-0.1, k_{I}=0.1, k_{D}=\right.$ $1.46406)$ to show the change of stability property caused by effective $W$ root classifications. These three points represent
Fig. 2. Complete real and effective $W$ root classifications for Example 2

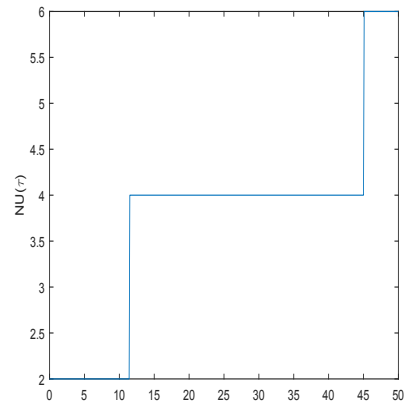

(a) At point " 1 "

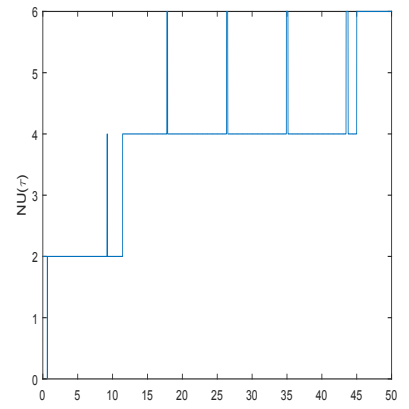

(b) At point " 3 "
Fig. 3. “ $N U(\tau)$ vs. $\tau$ ” plots for Example 2

the variation of $\left(k_{P}, k_{I}, k_{D}\right)$ near $\mathcal{N}_{W_{+}}^{\{2,1\}}$ as $k_{D}$ increases. At all of them $N U(0)=+2$, and hence the difference of stability property is due to the effective $W$ roots.

At point " 1 ", there is only one simple effective $W$ root 0.0350 and hence the closed-loop system has no stability $\tau$-interval. At point "2", there is one double effective $W$ root 0.5342 and one simple effective $W$ root 0.0350 . The double $W$ root is associated with a pair of degenerate CIRs $\lambda= \pm 0.7309 j$ : As $\tau$ increases near 0.6442 , a pair of conjugate characteristic roots touch without crossing the imaginary axis $\mathbb{C}_{0}$ at $\pm 0.7309 j$ in $\mathbb{C}_{+}$. This is a critical case that when $\tau=0.6442$, the closed-loop system has a pair of conjugate characteristic roots in $\mathbb{C}_{0}$ but no characteristic roots in $\mathbb{C}_{+}$. At point " 3 ", there are three simple effective $W$ roots 0.5379 , 0.5306 , and 0.0350 . As $\left(k_{P}, k_{I}, k_{D}\right)$ varies from point " 2 " to point " 3 ", the root loci touching the imaginary axis $\mathbb{C}_{0}$ (at point " 2 ") open a stability window (at point " 3 "): At point " 3 ", as $\tau$ increases near 0.64357 two conjugate characteristic roots cross $\mathbb{C}_{0}$ from right to left at $\pm 0.7284 j$ while as $\tau$ increases near 0.64472 two conjugate characteristic roots cross $\mathbb{C}_{0}$ from left to right at $\pm 0.7334 j$. The closed-loop system is asymptotically stable iff $\tau \in(0.64357,0.64472)$.

A $N U(\tau)$ comparison between points " 1 " and " 3 " is made in Fig. 3, where we see a structural change.

Example 3: Consider the plant (42) of [28],

$$
H_{0}(\lambda)=\frac{0.1(0.1 \lambda-1)(\lambda+0.1659)}{(\lambda-0.1081)\left(\lambda^{2}+0.2981 \lambda+0.06281\right)} .
$$




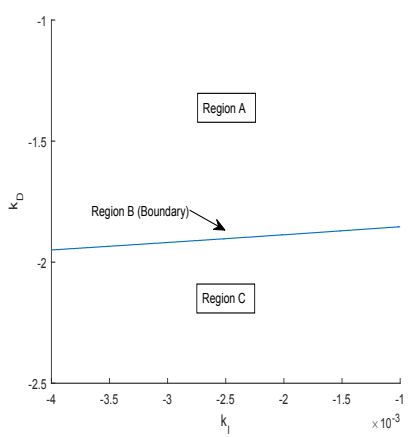

(a) Effective $W$ root classification

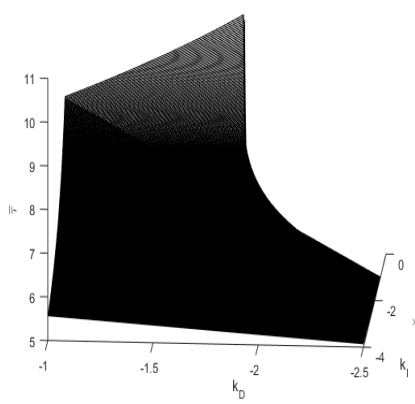

(b) Delay margin

Fig. 4. Complete effective $W$ root classification and delay margin for Example 3

According to the discussions in Subsection II-A, we adopt the PID controller with $-100<k_{D}<100$. We now use Procedure 1 for the complete effective $W$ root classification. For a clear illustration, we here choose a domain $\left(k_{I}, k_{D}\right) \in$ $[-0.004,-0.001] \times[-2.5,-1.0]$ with $k_{P}=-0.4143$.

The auxiliary characteristic function is $F_{\mathrm{PID}}(W)=$ $\chi_{4} W^{4}+\chi_{3} W^{3}+\chi_{2} W^{2}+\chi_{1} W+\chi_{0}$, where $\chi_{4}=1-\frac{k_{D}^{2}}{10000}$, $\chi_{3}=-\frac{k_{P}^{2}}{10000}+\frac{k_{I} k_{D}}{10000}-\left(\frac{10002752281 k_{D}}{1000000000000}-\frac{k_{I}}{10000}\right) k_{D}-\frac{1253539}{50000000}$, $\chi_{2}=-\frac{10002752281 k_{P}^{2}}{100000000000}-\left(\frac{2752281 k_{D}}{10000000000}-\frac{10002752281 k_{I}}{1000000000000}\right) k_{D}+$ $\left(\frac{10002752281 k_{D}}{1000000000000}-\frac{k_{I}}{10000}\right) k_{I}+\frac{35155752614521}{10000000000000000}$, $\chi_{1}=-\frac{2752281 k_{P}^{2}}{10000000000}+\frac{2752281 k_{D} k_{I}}{10000000000}+\left(\frac{2752281 k_{D}}{10000000000}-\right.$ $\left.10002752281 k_{I}\right) k_{I}+\frac{46100854437121}{1000000000000000}, \chi_{0}=-2752281 k_{I}^{2}$ $\left.\frac{10002752281 k_{I}}{1000000000000}\right) k_{I}+\frac{46100854437121}{1000000000000000000}, \chi_{0}=-\frac{2752281 k_{I}^{2}}{10000000000}$.

Then, by Steps 1 and 2 of Procedure 1, we may obtain the complete effective $W$ root classification. The selected domain may be divided into three regions (as depicted in Fig. 4(a)): Region A: $\mathcal{N}_{W_{+}}^{\{1\}}$; Region B: $\mathcal{N}_{W_{+}}^{\{2,1\}}$; Region C: $\mathcal{N}_{W_{+}}^{\{1,1,1\}}$.

For this example, the variation of the effective $W$ root classification also causes a distinct change for the stability set. We here display how $\bar{\tau}$ varies w.r.t. $k_{I}$ and $k_{D}$. Recall that $\bar{\tau}$ is the delay margin defined associated with (3), which is widely adopted as a stability/stabilization index in the literature. Using the methodology to be given later in this paper, we obtain the result as shown in Fig. 4(b). One may notice the discontinuity of $\bar{\tau}$ w.r.t. $\left(k_{I}, k_{D}\right)$. We now explain it from the angle of effective $W$ root classification. Choose a non-self-intersecting continuous path in Fig. 4(a) and let $\left(k_{I}, k_{D}\right)$ moves along this path from Region A to Region B (the boundary of Regions A and $\mathrm{C}$ ) and then to Region C. Some details are given below as $\left(k_{I}, k_{D}\right)$ is located in Regions $\mathrm{A}, \mathrm{B}$, and $\mathrm{C}$, respectively.

In Region A: As there is one simple effective $W$ root and $N U(0)=0$, the closed-loop system has one and only one stability $\tau$-interval. On Region B: As $\left(k_{I}, k_{D}\right)$ moves from Region A to Region B, an additional double effective $W$ root appears, which corresponds to a critical pair $(\lambda, \tau)$ with $\Delta N U_{\lambda}(\tau)=0$. For this example, it exhibits that, as $\left(k_{I}, k_{D}\right)$ moves from Region A to Region B, a breakpoint appears in the stability $\tau$-interval. In Region C: As $\left(k_{I}, k_{D}\right)$ moves from Region B to Region C, the double effective $W$ root splits into two distinct effective $W$ roots (the corresponding two set of critical pairs are with opposite signs for $\Delta N U_{\lambda}(\tau)$ ).

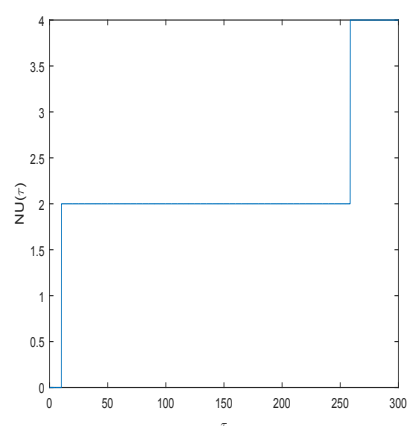

(a) $k_{D}=-1.8609$

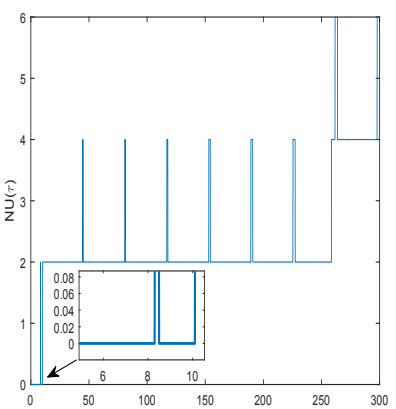

(b) $k_{D}=-1.8610$
Fig. 5. " $N U(\tau)$ vs. $\tau$ ” plots when $k_{I}=-0.0012$ for Example 3

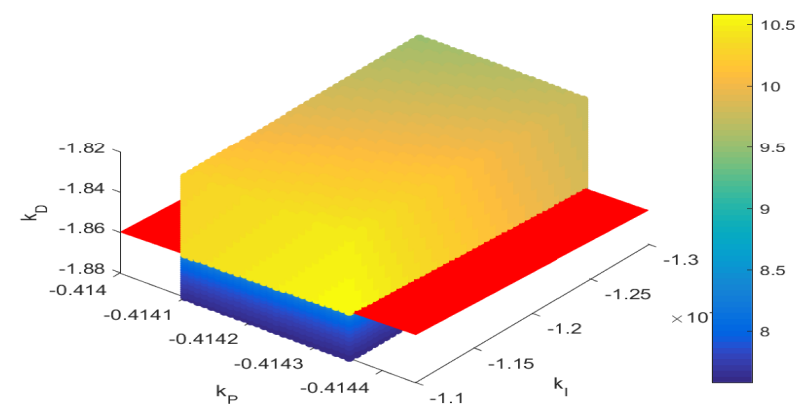

Fig. 6. Delay margin for Example 4

Consequently, as $\left(k_{I}, k_{D}\right)$ moves from Region B to Region C, the breakpoint expands and thereby the original one stability $\tau$-interval broke into two. This causes a discontinuity (more precisely, an abrupt decrease) of $\bar{\tau}$, as shown in Fig. 4(b).

We choose $\left(k_{I}=-0.0012, k_{D}=-1.8609\right)$ and $\left(k_{I}=\right.$ $\left.-0.0012, k_{D}=-1.8610\right)$ representing a point in Region A and a point in Region $\mathrm{C}$, both close to the boundary Region B. The " $N U(\tau)$ vs. $\tau$ " plots are given respectively in Fig. 5(a) and Fig. 5(b). It is seen that a very small change of $k_{D}$ causes a structural variation of the $N U(\tau)$ distribution.

In order to have a more intuitive illustration for the qualitative change mentioned in Example 3, we next consider the case where $k_{P}$ is also a free parameter near -0.4143 .

Example 4: For the system in Example 3, the stability set in the $\left(k_{P}, k_{I}, k_{D}, \tau\right)$-space will be studied in Example 8. We here specifically exhibit how the delay margin $\bar{\tau}$ varies w.r.t. $\left(k_{P}, k_{I}, k_{D}\right)$ in Fig. 6, where the color information represents $\bar{\tau}$ and the red surface is the boundary $\mathcal{N}_{W+}^{\{2,1\}}$. We can observe the discontinuity of $\tau$ ta sharp change in color from dark to light), which occurs exactly on the boundary $\mathcal{N}_{W+}^{\{2,1\}}$.

Remark 5: As illustrated in various examples in this paper, there may be more than one stability $\tau$-interval for the closedloop system with PID controller. By using our approach, all stability $\tau$-intervals can be precisely detected.

\section{FURTHER ALGEBRAIC INSIGHTS}

In this section, we will specifically reduce the associated computation burden by further taking into consideration the characteristics of the closed-loop system with PID controller. 


\section{A. Improved algebraic algorithm}

Theorem 2 requires the multiplicity information of the effective $W$ roots, and it is used at Step 5 of Algorithm 1.

As summarized in Remark 4, the $N U(\tau)$ distribution can be globally characterized in the $\left(k_{P}, k_{I}, k_{D}\right)$-space, in light of the complete effective $W$ root classification. In our methodology, Theorem 2 is used for a local analysis. In the sequel, we will present an improved version of Theorem 2.

For a controller vector $\left(k_{P}, k_{I}, k_{D}\right)$, label all the effective $W$ roots as $\widetilde{W}_{0}, \widetilde{W}_{1}, \ldots, \widetilde{W}_{\widetilde{u}-1}$ ( $\widetilde{u}$ denotes the number of effective $W$ roots, multiplicity taken into account) such that

$$
\widetilde{W}_{0} \geq \widetilde{W}_{1} \geq \widetilde{W}_{2} \geq \cdots
$$

For each $\widetilde{W}_{\alpha}$, we label a CIR $\widetilde{\lambda}_{\alpha}=j \widetilde{\omega}_{\alpha}\left(\widetilde{\omega}_{\alpha}^{2}=\widetilde{W}_{\alpha}\right)$ and denote the corresponding CDs by $\widetilde{\tau}_{\alpha, k}, k \in \mathbb{N}$.

Theorem 4: Consider the closed-loop system described by (1) and (2) with given $k_{P}, k_{I}$, and $k_{D}$. For any $\tau>0$ which is not a critical delay, $N U(\tau)$ can be explicitly expressed as

$$
N U(\tau)=N U(+\varepsilon)+\sum_{i=0}^{\widetilde{u}-1} \widetilde{N U}_{i}(\tau)
$$

where

$$
\begin{aligned}
& \widetilde{N U}_{i}(\tau)=\left\{\begin{array}{l}
0, \tau<\widetilde{\tau}_{i, 0}, \\
(-1)^{i} 2\left\lceil\frac{\tau-\widetilde{\tau}_{i, 0}}{2 \pi / \widetilde{\omega}_{i}}\right\rceil, \tau>\widetilde{\tau}_{i, 0},
\end{array} \quad \text { if } \widetilde{\tau}_{i, 0} \neq 0,\right. \\
& \widetilde{N U}_{i}(\tau)=\left\{\begin{array}{l}
0, \tau<\widetilde{\tau}_{i, 1}, \\
(-1)^{i} 2\left\lceil\frac{\tau-\widetilde{\tau}_{i, 1}}{2 \pi / \widetilde{\omega}_{i}}\right\rceil, \tau>\widetilde{\tau}_{i, 1},
\end{array} \quad \text { if } \widetilde{\tau}_{i, 0}=0 .\right.
\end{aligned}
$$

The proof of Theorem 4 is given in Appendix A.

By using Theorem 4, we may determine the expression of $N U(\tau)$ without knowing the multiplicity information of the effective $W$ roots.

Now, when employing Algorithm 1, Theorem 2 in Step 5 may be replaced by Theorem 4 . In this way, the multiplicity information concerning the effective $W$ roots is not explicitly required.

\section{B. Symmetry concerning effective $W$ roots}

First, it is not hard to see the following property for the coefficients of $F_{\mathrm{PID}}(W)$.

Property 5: The coefficients of the auxiliary characteristic function $F_{\mathrm{PID}}(W)$ (9) contain the terms $k_{P}^{2}, k_{I}^{2}, k_{D}^{2}$, and $k_{I} k_{D}$, without other terms involving $k_{P}, k_{I}$, or $k_{D}$.

Then, a useful result follows from Property 5:

Property 6: At a $\left(k_{P}, k_{I}, k_{D}\right)$-point, say $\left(\widehat{k}_{P}, \widehat{k}_{I}, \widehat{k}_{D}\right)$, all the $W$ roots are preserved if $\widehat{k}_{P}$ is replaced by $-\widehat{k}_{P}$ and/or $\left(\widehat{k}_{I}, \widehat{k}_{D}\right)$ is replaced by $\left(-\widehat{k}_{I},-\widehat{k}_{D}\right)$.

Thus, there is a symmetry concerning the effective $W$ roots. Without loss of generality, consider a domain of the form: $\left(k_{P}, k_{I}, k_{D}\right) \in[-a, a] \times([-b, 0) \cup(0, b]) \times[-c, c]$, where $a$, $b$, and $c$ are positive real numbers (recall that $k_{I} \neq 0$ in context of PID controller). We have the following result.

Property 7: For the closed-loop system described by (1) and (2), the complete real $W$ root classification for the domain $\left(k_{P}, k_{I}, k_{D}\right) \in[-a, a] \times([-b, 0) \cup(0, b]) \times[-c, c]$ can be

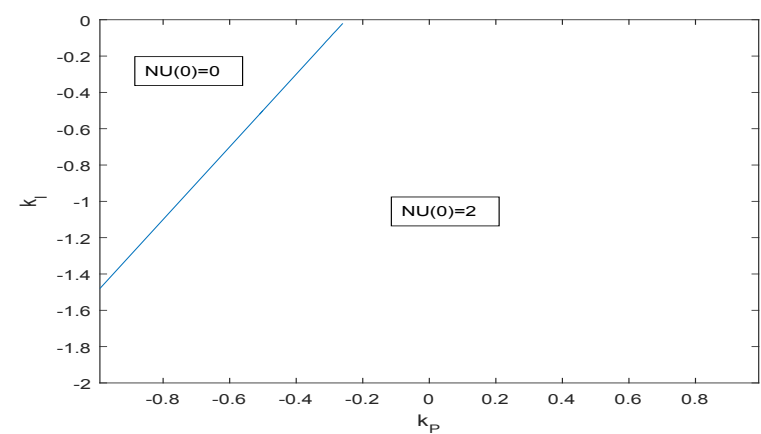

Fig. 7. $N U(0)$ vs. $\left(k_{P}, k_{I}\right)$ for Example 5

determined, if the complete real $W$ root classification for the domain $\left(k_{P}, k_{I}, k_{D}\right) \in[0, a] \times(0, b] \times[-c, c]$ is known.

Remark 6: Since a CIR $\lambda=j \omega$ is determined by $\omega^{2}=W$, all the CIRs of the closed-loop system are not changed if we replace $\widehat{k}_{P}$ by $-\widehat{k}_{P}$ and/or $\left(\widehat{k}_{I}, \widehat{k}_{D}\right)$ by $\left(-\widehat{k}_{I},-\widehat{k}_{D}\right)$. However, according to (8), the CDs are different.

Now, when employing Procedure 1, the domain to be examined for the complete real $W$ root classification in the $\left(k_{P}, k_{I}, k_{D}\right)$-space can be quartered, in light of Property 7.

\section{Methodology For EXAMINING STABILITy SET IN $\left(k_{P}, k_{I}, k_{D}, \tau\right)$-SPACE}

In this section, we will give a new parameter-space approach for examining the stability set, with which the PID stabilization problem can be systematically solved.

In the sequel, we preclude the trivial case where the closedloop system may be asymptotically stable independently of delay (the open-loop system is asymptotically stable, and the controller is not needed for the stabilization purpose).

\section{A. On $N U(+\varepsilon)$ analysis}

The value of $N U(+\varepsilon)$ is required by Theorem 2 as well as Theorem 4 . This calculation may be automatically finished by a computer if there is no CIR when $\tau=0$.

Recall that we denote the set where the closed-loop system has CIRs when $\tau=0$ by $\mathcal{N}_{W+}^{\tau=0}$. For a point in $\mathcal{N}_{W+}^{\tau=0}$, we may invoke the Puiseux series to determine the value of $N U(+\varepsilon)$ (according to Theorem 5.1 in [12] or Theorem 1 in [13]).

The set $\mathcal{N}_{W+}^{\tau=0}$ is in general with "measure 0 " in the $\left(k_{P}, k_{I}, k_{D}\right)$-space (special cases, if any, may be tested according to the complete effective $W$ root classification). In practice, we do not need to specifically analyze the set $\mathcal{N}_{W+}^{\tau=0}$. See the example below.

Example 5: Consider the controlled plant (41) in [28]:

$$
H_{0}(\lambda)=\frac{\lambda-2}{\lambda-0.5} .
$$

Following the discussions in Subsection II-A, the PID controller is not applicable and we should apply the PI controller (6) with $-1<k_{P}<1$. The $N U(0)$ values w.r.t. $k_{P}$ and $k_{I}$ are given in Fig. 7, where the boundary between the regions with $N U(0)=0$ and $N U(0)=2$ represents the set $\mathcal{N}_{W+}^{\tau=0}$. 
We first analyze the set $\mathcal{N}_{W+}^{\tau=0}$ by a standard method (through Theorem 5.1 in [12] or Theorem 1 in [13]). For a point $\left(k_{P}=-0.5, k_{I}=-0.5\right)$ on the boundary, the characteristic equation when $\tau=0, f(\lambda, 0)=0$, has a pair of CIRs $\sqrt{ } 2 j$. We may analyze the asymptotic behavior through invoking the Taylor series at the critical pair $(\sqrt{ } 2 j, 0) \dot{ } \Delta \lambda=(2.0000+$ $0.7071 j) \Delta \tau+o(\Delta \tau)$, which implies that $N U(+\varepsilon)=2$ at $\left(k_{P}\right.$ $\left.=-0.5, k_{I}=-0.5\right)$. By the continuity, the closed-loop system is not asymptotically stable for a sufficiently small $\tau$ on the boundary.

In fact, the above result may be easily derived from the stability set, shown in Fig. 8(b) (details will be given in Example 7). The border of the projection of the stability set on the $\left(k_{P}, k_{I}\right)$-plane is exactly the boundary in Fig. 7.

\section{B. New parameter-space approach}

Parameter-space approach (stability set in $\left(k_{P}, k_{I}, k_{D}, \tau\right)$ space):

Step 0: Select the controller type (PID or PI) according to the discussions in Subsection II-A.

Step 1: Obtain the complete effective $W$ root classification, by using Procedure 1.

Step 2: Sweep the $\left(k_{P}, k_{I}, k_{D}\right)$-space and for each $\left(k_{P}, k_{I}, k_{D}\right)$-point we study the complete stability problem using Algorithm 1.

Step 3: Display the stability set in the $\left(k_{P}, k_{I}, k_{D}\right)$-space. To be more precise: In the case of the PI controller, we display the stability set in the $\left(k_{P}, k_{I}, \tau\right)$-space. In the case of the PID controller, we display the stability set through one or multiple colored figures in the $\left(k_{P}, k_{I}, k_{D}\right)$-space. For a $\left(k_{P}, k_{I}, k_{D}\right)$ point, the stability range of $\tau$ can be expressed by one or multiple colors.

For a given PID controller vector $\left(k_{P}, k_{I}, k_{D}\right)$, if the stability $\tau$-set is non-empty, without loss of generality, we suppose that it is consisted of s stability intervals

$$
\tau \in\left(\underline{\tau}_{1}, \bar{\tau}_{1}\right) \cup \cdots \cup\left(\underline{\tau}_{\mathrm{s}}, \bar{\tau}_{\mathrm{s}}\right),
$$

plus possible $\tau=0$, with $0 \leq \underline{\tau}_{1}<\bar{\tau}_{1}<\cdots<\underline{\tau}_{\mathrm{s}}<\bar{\tau}_{\mathrm{s}}$. We call $\bar{\tau}_{\mathrm{s}}$ in (17) the generalized delay margin.

In the subsequent colored figures, we will display the generalized delay margin via the color information.

Remark 7: One may exhibit more information concerning the stability intervals in the form (17) through more colored points, without any technical difficulty. As a consequence, the stability set in the $\left(k_{P}, k_{I}, k_{D}, \tau\right)$-space may be displayed more comprehensively by multiple 3 -D figures.

We now present three useful corollaries for some frequentlyencountered cases. The explicit expression on generalized delay margin may be derived from these corollaries.

Corollary 2: If there is only one simple effective $W$ root with $\widetilde{\tau}_{0,0}>0$ and $N U(0)=0(N U(0)>0)$ in a region, then at each point in this region, the system is asymptotically stable iff $\tau \in\left[0, \widetilde{\tau}_{0,0}\right)$ (there is no stability $\tau$-interval).

Corollary 3: Suppose that there are two simple effective $W$ roots, with $\widetilde{\tau}_{0,0}>0$ and $\widetilde{\tau}_{1,0}>0$, and $N U(0)=0$ in a region. There are totally two possible cases at each point in this region:

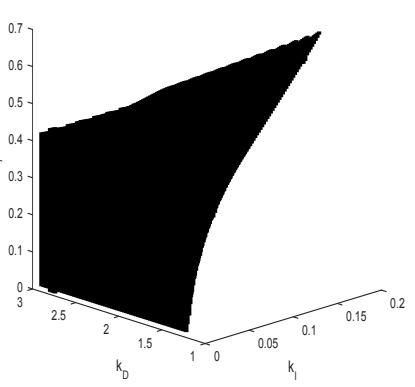

(a) Example 6

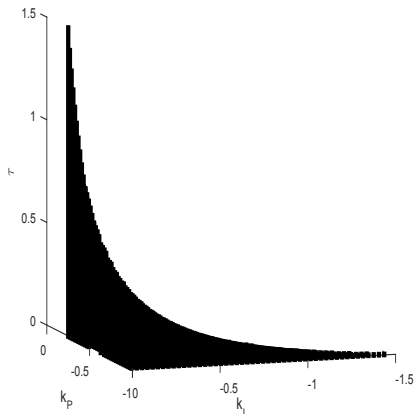

(b) Example 7
Fig. 8. Stability sets for Example 6 and Example 7

(1) If $\widetilde{\tau}_{1,0} \geq \widetilde{\tau}_{0,1}$, the system is asymptotically stable iff $\tau \in\left[0, \widetilde{\tau}_{0,0}\right)$.

(2) If $\widetilde{\tau}_{1,0}<\widetilde{\tau}_{0,1}$, there are multiple stability $\tau$-intervals: $\left[0, \widetilde{\tau}_{0,0}\right) \cup\left(\bigcup_{k=0}\left(\widetilde{\tau}_{1, k}, \widetilde{\tau}_{0, k+1}\right)\right)$ with $\mathrm{N}=\sup \left\{k: \widetilde{\tau}_{0, k}<\widetilde{\tau}_{1, k}<\right.$ $\left.\widetilde{\tau}_{0, k+1}\right\}$.

Corollary 4: Suppose that there are two simple effective $W$ roots, with $\widetilde{\tau}_{0,0}>0$ and $\widetilde{\tau}_{1,0}>0$, and $N U(0)=2$ in a region. There are totally three possible cases at each point in this region:

(1) If $\widetilde{\tau}_{1,0} \geq \widetilde{\tau}_{0,0}$, there is no stability $\tau$-interval.

(2) If $\widetilde{\tau}_{1,0}<\widetilde{\tau}_{0,0}$ and $\widetilde{\tau}_{1,1} \geq \widetilde{\tau}_{0,1}$, the system is asymptotically stable iff $\tau \in\left(\widetilde{\tau}_{1,0}, \widetilde{\tau}_{0,0}\right)$.

(3) Otherwise, there are multiple stability $\tau$-intervals: $\bigcup_{k=0}^{\mathrm{N}}\left(\widetilde{\tau}_{1, \mathrm{k}}, \widetilde{\tau}_{0, k}\right)$ with $\mathrm{N}=\sup \left\{k \geq 1: \widetilde{\tau}_{0,(k-1)}<\widetilde{\tau}_{1, k}<\right.$ $\left.\widetilde{\tau}_{0, k}^{k}\right\}$.

Based on the results of this paper, one may prove the above corollaries without any technical difficulty, .

For the second case in Corollary 3 and the second and the third cases in Corollary 4, the generalized delay margin is larger than the "classical" delay margin (the delay margin may be zero even if there exists a stability $\tau$-interval). For the other cases of the above Corollaries 2-4, the generalized delay margin and the delay margin are identical.

\section{ILLUSTRATIVE EXAMPLES}

In this section, we will give some examples to illustrate the proposed parameter-space approach.

Example 6: Using our parameter-space approach, we here examine the stability set for the system considered in Example 1, with $k_{P}=-0.1$. The result is given in Fig. 8(a).

In the stability set (Fig. 8(a)), $N U(0)$ may be positive. That is, the case where the plant can not be stabilized when $\tau=0$ is covered by our approach. Most of the stabilization approaches, e.g., [22] and [28], are not applicable in this case.

Remark 8: It is still a common technical limitation that "a time-delay system needs to be stabilizable when $\tau=0$ " in the existing stabilization studies. In addition, most of the existing results may be used to study only one stability $\tau$-interval in the form (3). These restrictions are removed in this paper. 


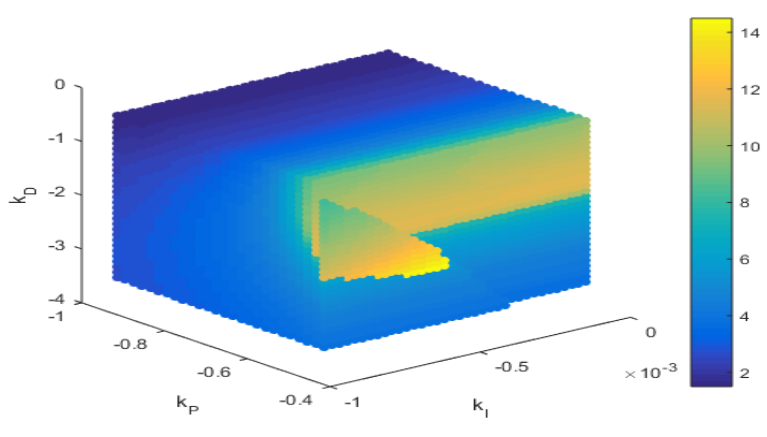

Fig. 9. Stability set for Example 8

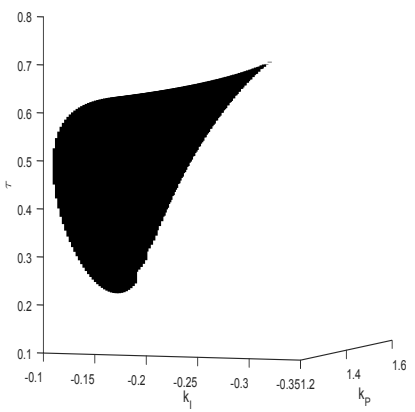

(a) PI controller

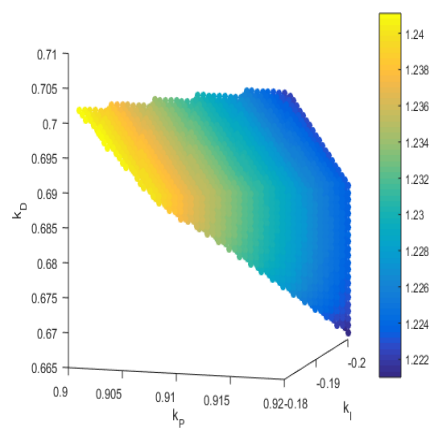

(b) PID controller
Fig. 10. Stability sets for Example 9

Example 7: For the controlled plant in Example 5, the lower stabilizable bound is about 1.5676 [28]. We now apply the parameter-space approach. As mentioned in Example 5, we employ the PI controller (6) with $-1<k_{P}<1$. The stability set is given in Fig. 8(b).

Example 8: Consider the controlled plant in Example 3. By using the approach of [28], two lower stabilizable bounds for $\tau$ are obtained: 15.4 and 12.6 with the following two required controllers respectively

$$
\begin{gathered}
\frac{-1.506 \times 10^{7}(\lambda+0.1307)\left(\lambda^{2}+0.2664 \lambda+0.0204\right)}{(\lambda+1103)(\lambda+418.7)(\lambda+3.376)(\lambda+0.1778)} \\
\times \frac{\left(\lambda^{2}+0.1582 \lambda+0.0303\right)\left(\lambda^{2}+0.2981 \lambda+0.0628\right)}{(\lambda+0.1659)(\lambda+0.1218)\left(\lambda^{2}+0.1885 \lambda+0.0333\right)}, \\
\frac{6.016 \times 10^{-5}(\lambda+0.275)\left(\lambda^{2}+0.2981 \lambda+0.0628\right)}{\lambda(\lambda+0.1659)(\lambda+10)\left(\lambda+0.083 \times 10^{6}\right)} .
\end{gathered}
$$

We now study the stability set under the PID controller, using the parameter-space approach. According to Step 0, we should employ the PID controller with $-100<k_{D}<100$. The stability set in the $\left(k_{P}, k_{I}, k_{D}, \tau\right)$-space is obtained as shown in Fig. 9. As mentioned, we use the color information to denote the generalized delay margin. It is worth mentioning that this system has more than one stability interval.

We find a controller vector $\left(k_{P}=-0.4143, k_{I}=\right.$ $\left.-0.0006, k_{D}=-2.3050\right)$ with the stability set $[0,5.4180) \cup$ $(14.3769,14.4952)$. That is, the maximum generalized delay margin is at least 14.4952 .

Example 9: Consider a controlled plant

$$
H_{0}(\lambda)=\frac{\lambda-\frac{1}{2}}{\left(\lambda-e^{\frac{\pi}{4} j}\right)\left(\lambda-e^{-\frac{\pi}{4} j}\right)}
$$

TABLE II

COMPUTATION TIME (SECONDS)

\begin{tabular}{|c|c|c|c|c|c|}
\hline & Fig. 8(a) & Fig. 8(b) & Fig. 9 & Fig. 10(a) & Fig. 10(b) \\
\hline Time & 0.071141 & 0.072166 & 4.209743 & 0.036372 & 1.202364 \\
\hline
\end{tabular}

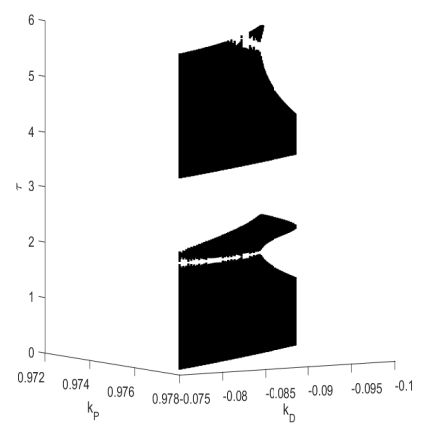

(a) Set with 3 and 4 stability $\tau$-intervals

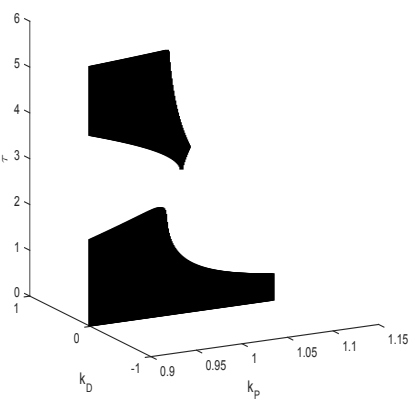

(b) Cross section $k_{D}=0$
Fig. 11. Stability set for Example 10

which is the controlled plant (45) of [28]. The lower stabilizable bound is about 0.55 [28]. Using the parameterspace approach, we obtain the stability sets under PI and PID controllers, as shown in Fig. 10(a) and Fig. 10(b). The maximum generalized delay margin is 0.7245 (1.2416) under the PI (PID) controller.

The computation time for obtaining the data of various figures in MATLAB (on a PC with an Intel Core $3.40 \mathrm{GHz}$ CPU with $32 \mathrm{G} \mathrm{RAM}$ ) is given in Table II. As we have mentioned, the parameter sweeping in the parameter-space approach is for a quantitative analysis of the $N U(\tau)$ distribution, and hence in practice we do not have to choose a very fine grid.

Finally, we give two examples with the PD controller

$$
k_{P}+k_{D} \lambda .
$$

The characteristic function of the closed-loop system with PD controller (18) reads

$$
f(\lambda, \tau)=H_{\mathrm{D}}(\lambda)+H_{\mathrm{N}}(\lambda)\left(k_{P}+k_{D} \lambda\right) e^{-\tau \lambda} .
$$

Our approach is applicable to this case.

Example 10: Consider the plant with $H_{\mathrm{D}}(\lambda)=\lambda^{5}+\left(\frac{\pi^{2}}{8}-\right.$ $\left.\frac{\pi}{2}+8\right) \lambda^{4}+\left(-\frac{\pi}{2}+3\right) \lambda^{3}+\left(\frac{\pi^{2}}{4}-\pi+10\right) \lambda^{2}+\left(-\frac{\pi}{2}+2\right) \lambda+$ $\frac{\pi^{2}}{8}-\frac{\pi}{2}+1$ and $H_{\mathrm{N}}(\lambda)=8 \lambda^{4}+\lambda^{3}+10 \lambda^{2}+\lambda+1$, which is borrowed from [11]. Here, we employ the PD controller (18).

First, following the discussions in Subsection II-A, we should confine $-\frac{1}{8}<k_{D}<\frac{1}{8}$. Then, using the parameterspace approach, we may obtain the stability set in the $\left(k_{P}, k_{D}, \tau\right)$-space. In some region, there are multiple stability intervals of $\tau$. Here, we specifically exhibit the part with three or four stability $\tau$-intervals in Fig. 11(a).

At $\left(k_{P}=1, k_{D}=0\right)$ there is a critical pair $(\lambda=j, \tau=\pi)$ with the Puiseux series $\Delta \lambda=0.1468 j(\Delta \tau)^{\frac{1}{2}}+(-0.0033-$ $0.1473 j)(\Delta \tau)^{\frac{2}{2}}+o\left((\Delta \tau)^{\frac{2}{2}}\right)$. It implies that at $\tau=\pi$, the CIR $\lambda=j$ is double and degenerate. More interestingly, the asymptotic behavior has a stabilizing effect: As $\tau$ increases 
near $\pi$, the appearance of this double CIR changes the stability property from being unstable to being asymptotically stable.

As the asymptotic behavior of CIRs in general case may be analyzed by our approach, the analysis for point $\left(k_{P}=\right.$ $\left.1, k_{D}=0\right)$ is included in the parameter-space approach, without any additional treatment. More precisely, for $\left(k_{P}=\right.$ $\left.1, k_{D}=0\right)$, there are three effective $W$ roots: $W_{0}=5.0268$ which is simple and with the critical pairs $(2.2421 j, 1.2525+$ $2.8024 k), W_{1}=1$ which is triple and with the critical pairs $(j,(2 k+1) \pi)$, and $W_{2}=0.1115$ which is simple and with the critical pairs $(0.3339 j, 5.8285+18.8155 k)$. By using Algorithm 1, we have that the closed-loop system has two and only two stability intervals of $\tau:[0,1.2525)$ and $(\pi, 4.0549)$. For a clear illustration, we give the cross section $k_{D}=0$ from the stability set in Fig. 11(b).

Remark 9: In characterizing the stability of time-delay systems, the asymptotic behavior analysis of multiple CIRs is a fundamental issue (see e.g., [3], [4], [11], [13], and [17]). The above example shows that the appearance of a multiple CIR may open a new stability interval of $\tau$. It is necessary to appropriately study the asymptotic behavior of multiple CIRs (otherwise, some stability set may be missing). As far as we know, none of the existing methods is valid for this case.

Finally, we consider the following second-order plant

$$
H_{0}(\lambda)=\frac{1}{\left(\lambda-p_{1}\right)\left(\lambda-p_{2}\right)} .
$$

Much attention has been paid to the stabilization of (20) subject to a delay $\tau$, in the following three cases (see e.g., [10], [15], [16], and [22]):

Case U.1: $p_{1}$ and $p_{2}$ are two positive real numbers.

Case U.2: $p_{1}$ and $p_{2}$ are a pair of conjugate complex numbers $\alpha \pm \beta j$ with $\alpha>0$.

Case U.3: $p_{1}$ and $p_{2}$ are a pair of conjugate imaginary numbers $\pm j \omega_{c}$.

In a very recent paper [15], the exact delay margin for (20) controlled by the PID controller is studied, for Cases U.1-U.3. It is pointed out therein that in order to achieve the maximal delay margin we should set $k_{I}=0$ (i.e., use the PD controller).

Example 11: We first consider Case U.1 for (20) and list the maximal delay margin and the maximal generalized delay margin in Table III. Cases U.2 and U.3 can be addressed in the same way. In particular, for Case U.3 many stability $\tau$ intervals can be found. For instance, if $p_{1}=j$ and $p_{2}=-j$, the maximal delay margin computed in [15] is 0.6198. Using our approach, we can find many stability $\tau$-intervals. If we choose $\left(k_{P}=0.01, k_{D}=0.01\right)$, it corresponds to the second case of Corollary 3 and there are thirty-six stability $\tau$-intervals and the generalized delay margin is 219.1508 . If we choose $\left(k_{P}=-0.01, k_{D}=-0.01\right)$, it corresponds to the third case of Corollary 4 and there are also thirty-six stability $\tau$-interv and the generalized delay margin is 222.2703 .

In [15], the following assumption is adopted:

Assumption 1: For the controlled plant (20), the controller gains of the PD controller (18) satisfy that $k_{P}>p_{1} p_{2}$ and $k_{D}>p_{1}+p_{2}$.

We now present a complete stability analysis of the secondorder plant (20) controlled by PD controller (18) using our
TABLE III

MAXIMAL DELAY MARGIN AND MAXIMAL GENERALIZED DELAY MARGIN

\begin{tabular}{|c|c|c|c|}
\hline & $\begin{array}{c}p_{1}=0.6 \\
p_{2}=0.8\end{array}$ & $\begin{array}{c}p_{1}=1 \\
p_{2}=1.2\end{array}$ & $\begin{array}{c}p_{1}=0.4 \\
p_{2}=2\end{array}$ \\
\hline $\begin{array}{c}\text { Maximal delay margin } \\
\text { by approach in [15] }\end{array}$ & 0.3960 & 0.2513 & 0.2595 \\
\hline $\begin{array}{c}\text { Maximal generalized delay margin } \\
\text { by approach in this paper }\end{array}$ & 0.8304 & 0.5304 & 0.4497 \\
\hline
\end{tabular}

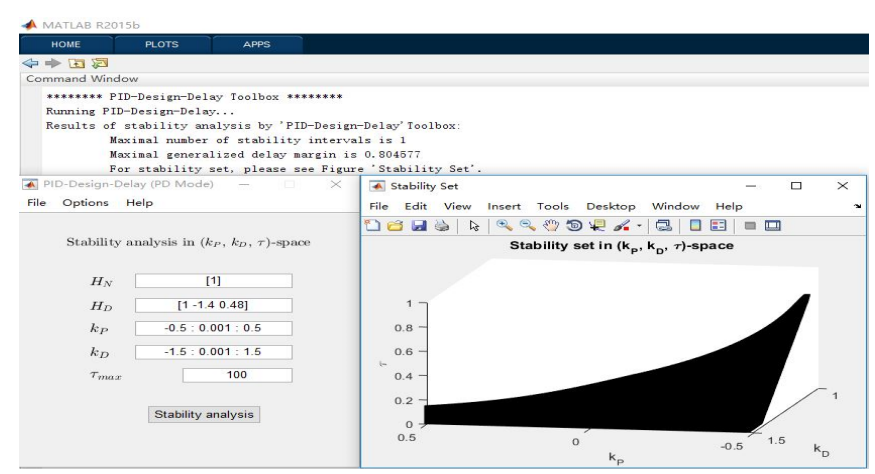

Fig. 12. Application of PID-Design-Delay toolbox

approach.

Proposition 2: Consider the closed-loop system described by (18) and (20). Under Assumption 1, for Cases U.1, U.2, and U.3, there is one and only one stability $\tau$-interval in the form (3) and the corresponding delay margin $\bar{\tau}$ is finitely large.

Proof: The characteristic function of the closed-loop system when $\tau=0$ is $f(\lambda, 0)=\lambda^{2}+\left(k_{D}-p_{1}-p_{2}\right) \lambda+k_{P}+p_{1} p_{2}$. Assumption 1 ensures that $k_{D}-p_{1}-p_{2}>0$ and $k_{P}+p_{1} p_{2}>0$, and hence both the two characteristic roots are in $\mathbb{C}_{-}$. Thus, $N U(0)=0$ in a parameter region where Assumption 1 holds.

The auxiliary characteristic function is $F_{\mathrm{PD}}(W)=W^{2}+$ $\left(p_{1}^{2}+p_{2}^{2}-k_{D}^{2}\right) W+p_{1}^{2} p_{2}^{2}-k_{P}^{2}$. Under Assumption $1, p_{1}^{2} p_{2}^{2}-$ $k_{P}^{2}<0$ and it is obvious that one $W$ root is positive real while the other one is negative real. Then, we have $\mathcal{N}_{W}^{\{1,1\}} \rightarrow$ $\mathcal{N}_{W}^{\left\{\frac{1}{1}, \overline{1}\right\}} \rightarrow \mathcal{N}_{W_{+}}^{\{1\}}$ for a region satisfying Assumption 1 .

We can now complete the proof by Corollary 2 .

According to Proposition 2, the maximal generalized delay margin equals to the maximal delay margin if Assumption 1 is imposed. More precisely, the value is exactly the maximal $\widetilde{\tau}_{0,0}$ in the region, in light of Corollary 2 .

Based on the parameter-space approach, we developed a MATLAB toolbox, PID-Design-Delay. It is freely available at http://faculty.neu.edu.cn/ise/lixuguang/PIDCDTDS.html.

The user only needs to input some requisite information through the graphical user interface ${ }^{1}$. The application to Example 11 is shown in Fig. 12.

\section{CONCLUdING REMARKS}

In this paper, the stabilization of time-delay systems using the proportional-integral-derivative (PID) controller is investigated. All the three controller gains $\left(k_{P}, k_{I}\right.$, and $\left.k_{D}\right)$ and the delay $(\tau)$ are treated as free parameters.

${ }^{1}$ With the manual and demo videos, a undergraduate can learn to design the PID controller and find the whole stability $\tau$-set in a couple of hours. 
First, under given $k_{P}, k_{I}$, and $k_{D}$, we develop an algebraic algorithm with which the complete stability analysis w.r.t. $\tau$ may be automatically performed through analyzing the $N U(\tau)$ distribution, where $N U(\tau)$ denotes the number of characteristic roots in the right-half plane as a function of $\tau$. As a result, the stability study in the $\left(k_{P}, k_{I}, k_{D}, \tau\right)$-space may be recast into the complete stability analysis w.r.t. $\tau$ in the $\left(k_{P}, k_{I}, k_{D}\right)$ space. Second, a procedure for the complete effective $W$ root classification ( $W$ is the auxiliary characteristic root) is proposed. With this classification, the $\left(k_{P}, k_{I}, k_{D}\right)$-space may be appropriately divided and all possible abrupt changes of the $N U(\tau)$ distribution can be accurately determined. Third, by specifically taking into account the characteristics of the closed-loop system with PID controller, the procedures for the above local and global analysis can be simplified.

Finally, we establish a new methodology (the new parameter-space approach) for determining the stability set in the $\left(k_{P}, k_{I}, k_{D}, \tau\right)$-space, which is systematic from both the theoretical and practical angles.

\section{ACKNOWLEDGMENT}

The authors wish to thank the Associate Editor and the anonymous reviewers for their constructive comments.

This work was supported in part by National Natural Science Foundation of China under Grant 61733003.

\section{APPENDIX A: PROOF OF THEOREM 4}

Note that we may label the effective $W$ roots in two forms: (10) and (15). Each $W_{i}^{o}\left(W_{i}^{e}\right)$ in (10) corresponds to $\mathcal{M}_{W_{i}^{o}}$ $\left(\mathcal{M}_{W_{i}^{e}}\right)$ elements in (15). Here, $\mathcal{M}_{W_{i}^{o}}\left(\mathcal{M}_{W_{i}^{e}}\right)$ denotes the multiplicity of $W_{i}^{o}\left(W_{i}^{e}\right)$, and hence $\mathcal{M}_{W_{i}^{o}}\left(\mathcal{M}_{W_{i}^{e}}\right)$ is an odd (even) number.

With the notations above, a CIR $\lambda_{i}^{o}\left(\lambda_{i}^{e}\right)$ is associated with $\mathcal{M}_{W_{i}^{o}}\left(\mathcal{M}_{W_{i}^{e}}\right)$ number of $\widetilde{\lambda}_{\alpha}$, the set for which is denoted by $\mathbb{S}_{\lambda_{i}^{o}}\left(\mathbb{S}_{\lambda_{i}^{e}}\right)$. Furthermore, for a $\lambda_{i}^{o}$, we denote the corresponding " $(-1)^{i}$ " in (11) by $U_{\lambda_{i}^{o}}$ and for a $\tilde{\lambda}_{\alpha}$, we denote the corresponding " $(-1)^{i}$ " in (16) by $U_{\widetilde{\lambda}_{\alpha}}$.

The following two properties are true according to the statement (2) of Theorem 1.

Property 8: If there exists at least one $W_{i}^{o}$ in (10), then $U_{\lambda_{0}^{o}}=+1$.

Property 9: If there is more than one $W_{i}^{o}$ in (10), then for any two consecutive $W_{i}^{o}$, say $W_{i^{\prime}}^{o}$ and $W_{i^{\prime \prime}}^{o}$, it follows that

$$
U_{\lambda_{i^{\prime}}^{o}} U_{\lambda_{i^{\prime \prime}}^{o}}=-1
$$

Our idea for proving Theorem 4, based on Theorem 2, is to show that the following two conditions always hold

$$
\begin{gathered}
U_{\lambda_{i}^{o}}=\sum_{\widetilde{\lambda}_{\alpha} \in \mathbb{S}_{\lambda_{i}^{o}}} U_{\widetilde{\lambda}_{\alpha}}, \\
0=\sum_{\widetilde{\lambda}_{\alpha} \in \mathbb{S}_{\lambda_{i}^{e}}} U_{\widetilde{\lambda}_{\alpha}} .
\end{gathered}
$$

The condition (22) is associated with a $\lambda_{i}^{o}$ (for which $U_{\lambda_{i}^{o}}=$ +1 or -1 ) and can be proved by Properties 8 and 9 . The condition (23), associated with a $\lambda_{i}^{e}$, is more obvious.

The proof of Theorem 4 is now complete.

\section{REFERENCES}

[1] B. Alikoç and A. F. Ergenç, "A new delay-independent stability test of LTI systems with single delay," 12th Workshop on Time Delay Systems, Ann Arbor, Michigan, USA, 2015.

[2] K. J. Åström and T. Hägglund, PID Controllers: Theory, Design, and Tuning. Research Triangle Park, NC, Instrument Society of America, 1995.

[3] I. Boussaada and S.-I. Niculescu, "Tracking the algebraic multiplicity of crossing imaginary roots for generic quasipolynomials: A Vandermondebased approach," IEEE Trans. Autom. Control, 61, 1601-1606, 2016.

[4] J. Chen, P. Fu, S.-I. Niculescu, and Z. Guan, "An eigenvalue perturbation approach to stability analysis, Part II: When will zeros of time-delay systems cross imaginary axis?" SIAM J. Contr. Optim., 48, 5583-5605, 2010.

[5] K. L. Cooke and P. van den Driessche, "On zeros of some transcendental equations," Funkcialaj Ekvacioj, 29, 77-90, 1986.

[6] I. I. Delice and R. Sipahi, "Delay-independent stability test for systems with multiple time-delays," IEEE Trans. Autom. Control, 57, 963-972, 2012.

[7] K. Gu, V. L. Kharitonov, and J. Chen, Stability of Time-Delay Systems. Boston, Birkhäuser, 2003.

[8] N. Hohenbichler, "All stabilizing PID controllers for time delay systems," Automatica, 45, 2678-2684, 2009.

[9] H. Y. Hu and Z. H. Wang, Dynamics of Controlled Mechanical Systems with Delayed Feedback. Heidelberg, Springer, 2002.

[10] P. Ju and H. Zhang, "Further results on the achievable delay margin using LTI control," IEEE Trans. Autom. Control, 61, 3134-3139, 2016.

[11] X. Li, J.-C. Liu, X.-G. Li, S.-I. Niculescu, and A. Çela, "Reversals in stability of linear time-delay systems: A finer characterization," Automatica, Article 108479, 2019.

[12] X.-G. Li, S.-I. Niculescu, and A. Çela, Analytic Curve FrequencySweeping Stability Tests for Systems with Commensurate Delays. London, Springer, 2015.

[13] X.-G. Li, S.-I. Niculescu, A. Çela, L. Zhang, and X. Li, "A frequencysweeping framework for stability analysis of time-delay systems," IEEE Trans. Autom. Control, 62, 3701-3716, 2017.

[14] X.-G. Li, L. Zhang, X. Li, and J.-X. Chen, "Colored stability crossing sets for SISO delay systems," IEEE Trans. Autom. Control, 63, 40164023, 2018

[15] D. Ma and J. Chen, "Delay margin of low-order systems achievable by PID controllers," IEEE Trans. Autom. Control, 64, 1958-1973, 2019.

[16] D. Ma, J. Chen, A. Liu, J. Chen, and S.-I. Niculescu, "Explicit bounds for guaranteed stabilization by PID control of second-order unstable delay systems," Automatica, 100, 407-411, 2019.

[17] W. Michiels, I. Boussaada, and S.-I. Niculescu, "An explicit formula for the splitting of multiple eigenvalues for nonlinear eigenvalue problems, and connections with the linearization for the delay eigenvalue problem," SIAM J. Matrix Anal. Appl., 38, 599-620, 2017.

[18] W. Michiels, "Spectrum based stability analysis and stabilization of systems described by delay differential algebraic equations," IET Control Theory and Applications, 5, 1829-1842, 2011.

[19] W. Michiels, K. Engelborghs, P. Vansevenant, and D. Roose, "The continuous pole placement method for delay equations," Automatica, 38 , 747-761, 2002.

[20] W. Michiels and S.-I. Niculescu, Stability, Control, and Computation for Time-Delay Systems: An Eigenvalue-Based Approach. Philadelphia, SIAM, 2014.

[21] W. Michiels, T. Vyhlídal, and P. Zítek, "Control design for timedelay systems based on quasi-direct pole placement," Journal of Process Control, 20, 337-343, 2010.

[22] R. H. Middleton and D. E. Miller, "On the achievable delay margin using LTI control for unstable plants," IEEE Trans. Autom. Control, 52, 1194-1207, 2007

[23] C.-I. Morărescu, C.-F. Méndez-Barrios, S.-I. Niculescu, and K. Gu, "Stability crossing boundaries and fragility characterization of PID controllers for SISO systems with I/O delays," American Control Conference, San Francisco, CA, USA, 2011.

[24] J. Neimark, "D-subdivisions and spaces of quasi-polynomials," Prikl. Mat. Meh., 13, 349-380, 1949.

[25] P. M. Nia and R. Sipahi, "Controller design for delay-independent stability of linear time-invariant vibration systems with multiple delays," J. Sound Vibr., 332, 3589-3604, 2013.

[26] N. Olgac and R. Sipahi, "An exact method for the stability analysis of time-delayed linear time-invariant (LTI) systems," IEEE Trans. Autom. Control, 47, 793-797, 2002. 
[27] L.-L. Ou, W.-D. Zhang, and L. Yu, "Low-order stabilization of LTI systems with time delay," IEEE Trans. Autom. Control, 54, 774-787, 2009.

[28] T. Qi, J. Zhu, and J. Chen, "Fundamental limits on uncertain delays: When is a delay system stabilizable by LTI controllers?" IEEE Trans. Autom. Control, 62, 1314-1328, 2017.

[29] G. J. Silva, A. Datta, and S. P. Bhattacharyya, PID Controllers for TimeDelay Systems. Boston, Birkhäuser, 2005.

[30] K. Walton and J. E. Marshall, "Direct method for TDS stability analysis," Proc. IEE, Part D, 134, 101-107, 1987.

[31] H. Wang, J. Liu, and Y. Zhang, "New results on eigenvalue distribution and controller design for time delay systems," IEEE Trans. Autom. Control, 62, 2886-2901, 2017.

[32] Z. H. Wang and H. Y. Hu, "Stability switches of time-delayed dynamic systems with unknown parameters," J. Sound Vibr., 233, 215-233, 2000.

[33] L. Yang, "Recent advances on determining the number of real roots of parametric polynomials," J. Symb. Comput., 28, 225-242, 1999. 